\title{
The Prognosis of Single Large Hepatocellular Carcinoma Was Distinct from Barcelona Clinic Liver Cancer Stage A or B: The Role of Albumin-Bilirubin Grade
}

\author{
Kuan-Chieh Fang ${ }^{a, b} \quad$ Wei-Yu Kao ${ }^{a-e} \quad$ Chien-Wei Su ${ }^{a, c}$ Po-Chun Chen ${ }^{a}$ \\ Pei-Chang Lee ${ }^{a, c}$ Yi-Hsiang Huang ${ }^{a, f}$ Teh-la Huo a,g Chun-Chao Chang ${ }^{b, d}$ \\ Ming-Chih Hou ${ }^{a}, \mathrm{c}, \mathrm{h}$ Han-Chieh Lin ${ }^{\mathrm{a}, \mathrm{c}}$ Jaw-Ching Wu ${ }^{\mathrm{f}, \mathrm{i}}$ \\ ${ }^{a}$ Division of Gastroenterology and Hepatology, Department of Medicine, Taipei \\ Veterans General Hospital, Taipei, Taiwan; ${ }^{b}$ Division of Gastroenterology and \\ Hepatology, Department of Internal Medicine, Taipei Medical University Hospital, \\ Taipei, Taiwan; ' Faculty of Medicine, School of Medicine, National Yang-Ming University, \\ Taipei, Taiwan; d Division of Gastroenterology and Hepatology, Department of Internal \\ Medicine, School of Medicine, College of Medicine, Taipei Medical University, \\ Taipei, Taiwan; ${ }^{\text {e } G r a d u a t e ~ I n s t i t u t e ~ o f ~ C l i n i c a l ~ M e d i c i n e, ~ C o l l e g e ~ o f ~ M e d i c i n e, ~ T a i p e i ~}$ \\ Medical University, Taipei, Taiwan; ${ }^{f}$ Institute of Clinical Medicine, School of Medicine, \\ National Yang-Ming University, Taipei, Taiwan; ${ }^{9}$ Department and Institute of Pharmacology,

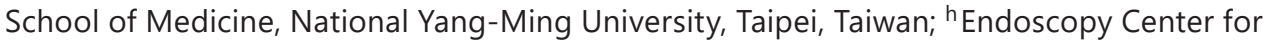 \\ Diagnosis and Treatment, Taipei Veterans General Hospital, Taipei, Taiwan; 'Department of \\ Medical Research, Taipei Veterans General Hospital, Taipei, Taiwan
}

\section{Keywords}

Barcelona Clinic Liver Cancer stage $\cdot$ Hepatocellular carcinoma · Prognosis $\cdot$ Staging $\cdot$ Tumor size

\begin{abstract}
Background/Aims: Whether single large hepatocellular carcinoma (SLHCC) is classified as Barcelona Clinic Liver Cancer (BCLC) stage A or B is still controversial. We aimed to compare the clinical manifestations, treatment modalities, and prognoses among patients with SLHCC and those in BCLC stage A and B. Methods: We enrolled 2,285 treatment-naive hepatocellular carcinoma (HCC) patients with BCLC stage A or B from October 2007 to December 2015. Factors in terms of prognoses were analyzed by multivariate analysis. Results: We enrolled 1,210, 466 , and 609 patients in a BCLC-A, SLHCC, and BCLC-B group, respectively. After a median follow-up duration of 21.2 months, 898 patients had died. The cumulative 5 -year survival rates
\end{abstract}

Part of this study was presented as a poster at the 52nd annual meeting of the European Association for the Study of the Liver, Amsterdam, Netherlands, April 19-23, 2017. 
Fang et al.: Single Large Hepatocellular Carcinoma

were $57.0,42.6$, and $27.3 \%$ for patients in the BCLC-A, SLHCC, and BCLC-B groups, respectively, which were significantly different $(p<0.001)$. Multivariate analysis indicated that the following independent risk factors were associated with poor prognosis: age $>65$ years, alkaline phosphatase $>100 \mathrm{U} / \mathrm{L}$, creatinine $>1.0 \mathrm{mg} / \mathrm{dL}$, alpha-fetoprotein $>20 \mathrm{mg} / \mathrm{mL}$, noncurative treatment, albumin-bilirubin (ALBI) grade, and HCC staging. Subgroup analysis also confirmed that patients in the SLHCC group had a survival rate intermediate to those in the BCLC$A$ and BCLC-B groups. However, for patients in the SLHCC group and with ALBI grade 1, outcomes were close to those in the BCLC-A group, especially in the setting of curative treatment. For those with ALBI grades 2 or 3, the prognoses were similar to those of the SLHCC and BCLC$B$ groups. Conclusion: Patients in the SLHCC group had an overall survival rate intermediate to those of the BCLC-A and BCLC-B groups. It is suggested that the SLHCC group could be classified as occupying a different stage from the BCLC stages $A$ and $B$. The ALBI grade could help to stratify SLHCC into a different prognostic group. However, the results need to be validated externally in other regions of the world.

(C) 2018 S. Karger AG, Basel

\section{Introduction}

Hepatocellular carcinoma (HCC) is the fifth most common malignancy and ranks as the second and sixth leading cause of cancer death in males and females, respectively. Worldwide approximately 700,000 people die of HCC annually [1]. The prognosis of patients with HCC has improved due to the promotion of HCC surveillance programs for high-risk groups, more cases being diagnosed and treated at early stages, and recent advances in treatments [2-4]. However, outcomes are still suboptimal. One recent study conducted in the United States showed that the 5-year overall survival (OS) rates for patients with liver cancer increased from 3\% between 1975 and 1977 to 18\% between 2004 and 2010 [5]. Evidence-based staging and management of HCC is the cornerstone in achieving an appropriate care in clinical practice and improving the prognosis of patients. The Barcelona Clinic Liver Cancer (BCLC) staging system is endorsed by the American Association for the Study of Liver Diseases (AASLD) and the European Association for the Study of the Liver (EASL) [6] and has been widely applied to guide the management of patients with HCC and to predict their prognoses [7, 8].

For patients with single large ( $>5 \mathrm{~cm}$ in size) HCC (SLHCC) with good liver functional reserve and performance status and without vascular invasion, liver transplantation is not recommended, and local ablation therapies are applied less due to the large tumor burden. Instead, resection surgery or transarterial chemoembolization (TACE) may be the first-line treatment in this clinical setting [9]. However, their optimal staging and treatment modalities are not fully elucidated. The original version of the BCLC staging system, which was published in 1999, defined BCLC stage A HCC as single tumors, or two or three tumors $<3 \mathrm{~cm}$, that were suitable for radical therapies. Thus, SLHCC is categorized as BCLC stage A in this setting [10]. Nevertheless, in 2002, Bruix and Llovet [11] remarked that "patients at an early stage are those who present asymptomatic single HCC $\leq 5 \mathrm{~cm}$ or up to 3 nodules $\leq 3 \mathrm{~cm}$." By this statement, SLHCC may be classified as BCLC stage B. Furthermore, according to the updated BCLC staging system (2010), "early stage HCC (BCLC A) classification consists of patients with single HCC or with up to three nodules $<3 \mathrm{~cm}$," and "intermediate stage (BCLC B) is formed by those patients with single large HCCs and those with multifocal disease who are asymptomatic and do not present vascular invasion or extrahepatic spread." By this definition, SLHCC may be stage A or stage B [12]. Several studies have been conducted to investigate the prognosis of patients with SLHCC to determine the most appropriate BCLC staging for SLHCC; however, the results are conflicting [13-17]. Therefore, the BCLC classification of SLHCC 


\section{Liver Cancer}

Fig. 1. Study flowchart. BCLC, Barcelona Clinic Liver Cancer; HCC, hepatocellular carcinoma; LT, liver transplantation; RFA, radiofrequency ablation; SLHCC, single large hepatocellular carcinoma; TACE, transarterial chemoembolization.

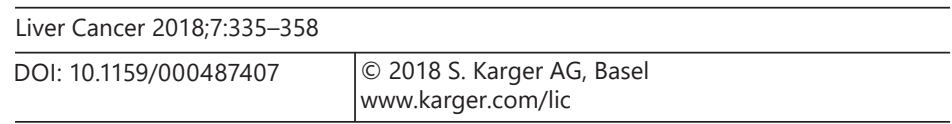

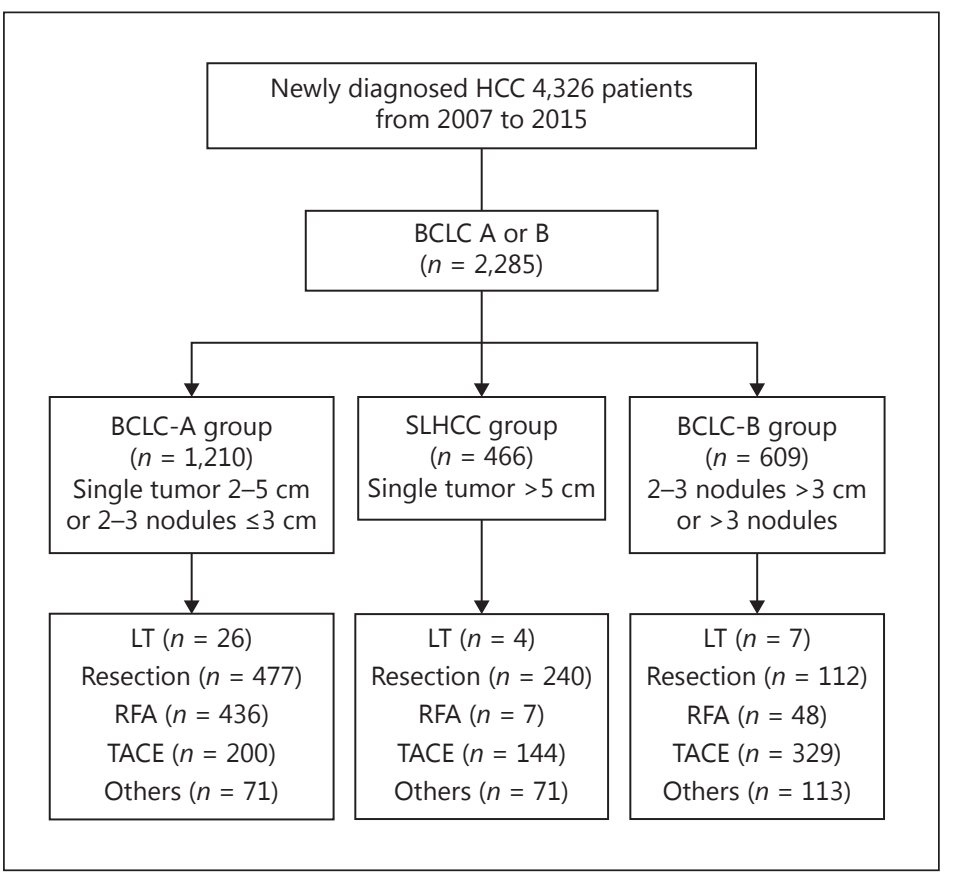

remains controversial [18]. This may cause ambiguities in cancer registries and result in communication gaps among researchers and clinical studies.

The majority of patients with HCC have underlying advanced chronic liver disease or even portal hypertension [19], which could make the adoption of treatment strategies and the prediction of outcomes more complex. In addition to the tumor factors, the grade of liver function is also crucial in determining the outcomes of HCC patients, especially of those with early-stage tumor [20]. These factors should be carefully assessed at the time of HCC diagnosis to determine the appropriate treatment and to predict the prognosis. Among the assessments of liver functional reserve, the albumin-bilirubin (ALBI) score, including both serum albumin and bilirubin levels, has been proposed and validated to provide a simple and objective method to predict the outcome of patients with HCC $[21,22]$. However, the application of the ALBI score in predicting the outcomes of patients with SLHCC has not yet been well studied. We hypothesized that liver functional reserve would determine the prognosis of patients with SLHCC and that ALBI may help to categorize SLHCC into a more suitable BCLC staging. To test these hypotheses, we analyzed a large cohort of HCC patients to compare the clinical manifestations, treatment modalities, and prognoses in patients with SLHCC and those in BCLC stage A and B. Using this analysis, we also aimed to identify the optimal BCLC staging of SLHCC.

\section{Patients and Methods}

Patients and Follow-Up

This cohort study was prospectively conducted and retrospectively analyzed. We enrolled 4,326 consecutive treatment-naive patients who fulfilled the diagnostic criteria of HCC by the AASLD consensus [23]. These were included in the cancer registration system at the Taipei Veterans General Hospital from October 2007 to December 2015. All of the patients were followed up every 3 months until their last visit to the hospital, death, or July 16, 2016. All patients who were newly diagnosed with HCC at Taipei Veterans General Hospital were assessed to determine a diagnosis and treatment strategy in a multidisciplinary committee. 
Fang et al.: Single Large Hepatocellular Carcinoma

The decision of treatment modality was shared with the patient and the physician by the multidisciplinary experts after discussing the risks, benefits, complications, and efficacies of the currently available treatments. The choice of treatment is mainly based on the stage of HCC, severity of the underlying liver disease, performance status, availability of treatment resources, and clinical expertise by the AASLD and EASL guidelines [8]. All of the demographic characteristics, clinicopathology, treatments, and outcomes of the enrolled patients were prospectively collected in the database of our multidisciplinary committee.

A total of 2,285 patients with BCLC stage A or B HCC were enrolled for the final analysis after excluding patients without complete data for the BCLC stage and those in BCLC stage 0, C, or D (Fig. 1). The BCLC-A group was defined as patients having a single tumor $2-5 \mathrm{~cm}$ in size or two or three nodules $\leq 3 \mathrm{~cm}$ in size. The SLHCC group included patients with a single tumor $>5 \mathrm{~cm}$ in size. The BCLC-B group was defined as patients with two or three nodules $>3 \mathrm{~cm}$ in size or $>3$ nodules. The number of patients was 1,210, 466, and 609 in the BCLC-A, SLHCC, and BCLC-B group, respectively.

The numbers of patients undergoing curative treatment including liver transplantation, resection surgery, and radiofrequency ablation were 37, 829, and 491, respectively, and the numbers of patients undergoing noncurative treatment of TACE or other treatments (such as best supportive treatment, chemotherapy, sorafenib, radiotherapy, and chemoradiotherapy) were 673 and 255 , respectively.

\section{Biochemical and Serologic Markers}

Serum hepatitis B virus surface antigen and hepatitis $\mathrm{C}$ virus antibody were tested by radioimmunoassay (Abbott Laboratories, North Chicago, IL, USA) and second-generation enzyme immunoassay (Abbott Laboratories). Serum biochemistry was measured using a Roche/Hitachi Modular Analytics System (Roche Diagnostics GmbH, Mannheim, Germany). Serum alpha-fetoprotein (AFP) level was tested using a radioimmunoassay kit (Serono Diagnostic SA, Coinsins, Switzerland). The ALBI score was calculated using the formula $(-0.085 \times$ albumin in $\mathrm{g} / \mathrm{L})+\left(0.66 \times \log _{10}\right.$ bilirubin in $\left.\mu \mathrm{mol} / \mathrm{L}\right)$ [21]. ALBI grades were defined as grade 1 (score $\leq-2.60$ ), grade 2 (score $>-2.60$ and $\leq-1.39$ ), and grade 3 (score $>-1.39$ ).

\section{Statistical Analysis}

The primary endpoint of this study was OS. This parameter was calculated from the diagnosis of HCC to death, the last visit, or loss to follow-up. The baseline characteristics and outcomes were selected according to the EASL guidelines [6]. Pearson $\chi^{2}$ analysis or the Fisher exact test was performed to compare categorical variables, and the Kruskal-Wallis test or the Mann-Whitney U test was applied to compare continuous variables between two or more patient groups. Cumulative OS rates were estimated using the Kaplan-Meier method and compared using the Cox proportional hazards model. In addition, we confirmed the assumption of proportional hazards using the log-minus-log plot of survival in a Cox regression analysis. Variables that reached statistical significance $(p<0.05)$ or came near statistical significance $(p<0.1)$ after univariate analysis underwent multivariate analysis via a forward stepwise Cox regression model. A two-tailed $p$ value $<0.05$ was considered to be statistically significant. All statistical analyses were performed using IBM SPSS Statistics for Windows, version 21.0 (IBM Corp., Armonk, NY, USA).

\section{Results}

\section{Demographic Data}

The baseline demographic characteristics of the enrolled patients are presented in Table 1. There were significant differences in the distribution of sex, serum biochemistry measures, viral factors, tumor factors, liver functional reserve (such as ALBI grade and presence of esophageal varices), and treatment modalities in the three patient groups.

\section{OS of Patients in the BCLC-A, SLHCC, and BCLC-B Groups}

After a median follow-up of 21.2 months (interquartile range 8.0-43.8 months), 898 patients had died and 1,387 were still alive. The cumulative $0 S$ rates at 1,3 , and 5 years were 89.2, 70.1, and $57.0 \%$ in the BCLC A group, 75.0, 54.5, and $42.6 \%$ in the SLHCC group, and $66.3,40.5$, and $27.3 \%$ in the BCLC B group, respectively. Statistically significant differences in the OS rates were noted in the pairwise comparison across all three groups (all $p<0.001$; 
Fang et al.: Single Large Hepatocellular Carcinoma

Table 1. Baseline demographics of the enrolled HCC patients

\begin{tabular}{|c|c|c|c|c|}
\hline Variable & BCLC A $(n=1,210)$ & SLHCC $(n=466)$ & BCLC B $(n=609)$ & $p$ \\
\hline \multicolumn{5}{|l|}{ Patient demographics } \\
\hline Age, years & $67(57-75)$ & $69(57-79)$ & $67(58-76)$ & 0.200 \\
\hline Male sex & $882(72.9 \%)$ & $375(80.5 \%)$ & $460(75.5 \%)$ & 0.005 \\
\hline \multicolumn{5}{|c|}{ Serum biochemistry tests and liver function tests } \\
\hline Albumin, g/dL & $3.90(3.40-4.20)$ & $3.80(3.40-4.10)$ & $3.60(3.10-4.00)$ & 0.787 \\
\hline Total bilirubin, $\mathrm{mg} / \mathrm{dL}$ & $0.77(0.53-1.16)$ & $0.73(0.53-1.12)$ & $0.80(0.55-1.21)$ & 0.554 \\
\hline Alanine aminotransferase, $\mathrm{U} / \mathrm{L}^{1}$ & $39(26-63)$ & $42(26-63)$ & $47(30-77)$ & 0.074 \\
\hline Aspartate aminotransferase, $\mathrm{U} / \mathrm{L}^{1}$ & $41(28-68)$ & $47(32-86)$ & $57(38-94)$ & $<0.001$ \\
\hline Creatinine, $\mathrm{mg} / \mathrm{dL}^{1}$ & $0.92(0.78-1.13)$ & $0.94(0.77-1.14)$ & $0.94(0.78-1.18)$ & 0.421 \\
\hline Alkaline phosphatase, $\mathrm{U} / \mathrm{L}^{1}$ & $80(63-106)$ & $96(73-131.75)$ & $108(79-155)$ & $<0.001$ \\
\hline \multirow[t]{2}{*}{ Platelets, $/ \mathrm{mm}^{3}$} & 131,000 & 187,000 & 143,000 & $<0.001$ \\
\hline & $(86,000-177,000)$ & $(136,000-240,000)$ & $(101,000-210,000)$ & \\
\hline $\mathrm{PT} / \mathrm{INR}^{1}$ & $1.07(1.01-1.14)$ & $1.06(1.01-1.11)$ & $1.08(1.03-1.17)$ & 0.631 \\
\hline \multicolumn{5}{|l|}{ Viral factors } \\
\hline $\operatorname{HBsAg}(+/-)^{1}$ & $599 / 452(57.0 / 43.0 \%)$ & $228 / 203(52.9 / 47.1 \%)$ & $284 / 272(51.1 / 48.9 \%)$ & 0.058 \\
\hline Anti-hepatitis $C$ virus $(+/-)^{1}$ & $380 / 605(38.6 / 61.4 \%)$ & $84 / 336(20 / 80 \%)$ & $188 / 364(34.1 / 64.5 \%)$ & $<0.001$ \\
\hline Antiviral therapy (yes/no) & $359 / 851(29.7 / 70.3 \%)$ & $54 / 412(11.6 / 88.4 \%)$ & $91 / 518(14.9 / 85.1 \%)$ & $<0.001$ \\
\hline \multicolumn{5}{|l|}{ Tumor factors } \\
\hline Tumor size, $\mathrm{cm}$ & $2.8(2.3-3.5)$ & $8.0(6.5-11.13)$ & $5.10(3.50-8.20)$ & $<0.001$ \\
\hline Tumor number (single/multiple) & $942 / 268(77.9 / 22.1 \%)$ & $466 / 0(100 / 0 \%)$ & $0 / 609(0 / 100 \%)$ & $<0.001$ \\
\hline Alpha-fetoprotein, $\mathrm{ng} / \mathrm{mL}^{1}$ & $16.71(5.53-89.11)$ & $39.14(5.23-1438.74)$ & $62.50(9.48-928.52)$ & $<0.001$ \\
\hline EVs $(+/-)^{1}$ & $215 / 908(19.1 / 80.7 \%)$ & $43 / 376(10.3 / 89.7 \%)$ & $96 / 429(18.3 / 81.7 \%)$ & $<0.001$ \\
\hline ALBI grade $(1 / 2 / 3)$ & $\begin{array}{l}539 / 583 / 88 \\
(44.5 / 48.2 / 7.3 \%)\end{array}$ & $\begin{array}{l}197 / 236 / 33 \\
(42.3 / 50.6 / 7.1 \%)\end{array}$ & $\begin{array}{l}197 / 324 / 88 \\
(32.3 / 53.2 / 14.5 \%)\end{array}$ & $<0.001$ \\
\hline Curative/noncurative treatment & $939 / 271(77.6 / 22.4 \%)$ & $251 / 215(53.9 / 46.1 \%)$ & $167 / 442(27.4 / 72.6 \%)$ & $<0.001$ \\
\hline
\end{tabular}

Continuous variables are expressed as median with 25th and 75th percentiles. ALBI, albumin-bilirubin; BCLC, Barcelona Clinic Liver Cancer; EVs, esophageal varices; HBsAg, hepatitis B virus surface antigen; HCC, hepatocellular carcinoma; PT/INR, prothrombin time/ international normalized ratio; SLHCC, single large hepatocellular carcinoma. ${ }^{1}$ Missing data at the time of HCC diagnosis in this parameter.

Fig. 2a), and the results were similar after excluding patients who underwent liver transplantation (all $p<0.001$; Fig. 2b).

We compared the prognoses among the three groups using subgroup analysis. In all subgroups except for female patients, the OS rates were significantly higher in the BCLC-A group than in the SLHCC group (Fig. 2c). Similarly, the OS rates were higher in the SLHCC group than in the BCLC-B group in all subgroups except for those with anti-hepatitis $C$ virus positivity, with antiviral therapy, with presence of esophageal varices, or with a prothrombin time/international normalized ratio $>1.1$ (Fig. 2d). The OS rates were significantly higher in the BCLC-A group than those in BCLC-B group in all the subgroups (Fig. 2e).

\section{Multivariate Analysis of Independent Risk Factors Associated with Poor Prognosis}

Since the ALBI scores were calculated using serum albumin and bilirubin levels, we applied two multivariate analysis models. In model 1, the ALBI grade was enrolled, but serum albumin and bilirubin levels were not entered into the multivariate analysis. In model 2 , we selected serum albumin and bilirubin levels, but not the ALBI grade in the multivariate analysis.

As shown in Table 2, in model 1, age $>65$ years, serum alkaline phosphatase levels $>100 \mathrm{U} / \mathrm{L}$, creatinine levels $>1.0 \mathrm{mg} / \mathrm{dL}$, AFP levels $>20 \mathrm{mg} / \mathrm{mL}$, noncurative treatment, higher ALBI grade, and HCC group were the independent risk factors associated with poor OS. 


\section{Liver Cancer}

Fig. 2. a Comparison of cumulative OS rates among BCLC stage A, SLHCC, and BCLC stage B stratified by HCC stage. b The same analysis after excluding patients who underwent liver transplantation. c-e Comparison of OS among the three groups of patients in stratified analysis by forest plot: BCLC stage A versus SLHCC (c), SLHCC versus BCLC stage B (d), and BCLC stage A versus stage $B$ (e). AFP, alpha-fetoprotein; ALP, alkaline phosphatase; ALT, alanine aminotransferase; AST, aspartate aminotransferase; BCLC, Barcelona Clinic Liver Cancer; EVs, esophageal varices; HBsAg, hepatitis B virus surface antigen; HCC, hepatocellular carcinoma; HCV, hepatitis C virus; OS, overall survival; PT/INR, prothrombin time/international normalized ratio; SLHCC, single large hepatocellular carcinoma.

\begin{tabular}{l|l}
\hline Liver Cancer 2018;7:335-358 \\
\hline DOI: 10.1159/000487407 & $\begin{array}{l}\text { (c) 2018 S. Karger AG, Basel } \\
\text { www.karger.com/lic }\end{array}$ \\
\hline
\end{tabular}

Fang et al.: Single Large Hepatocellular Carcinoma

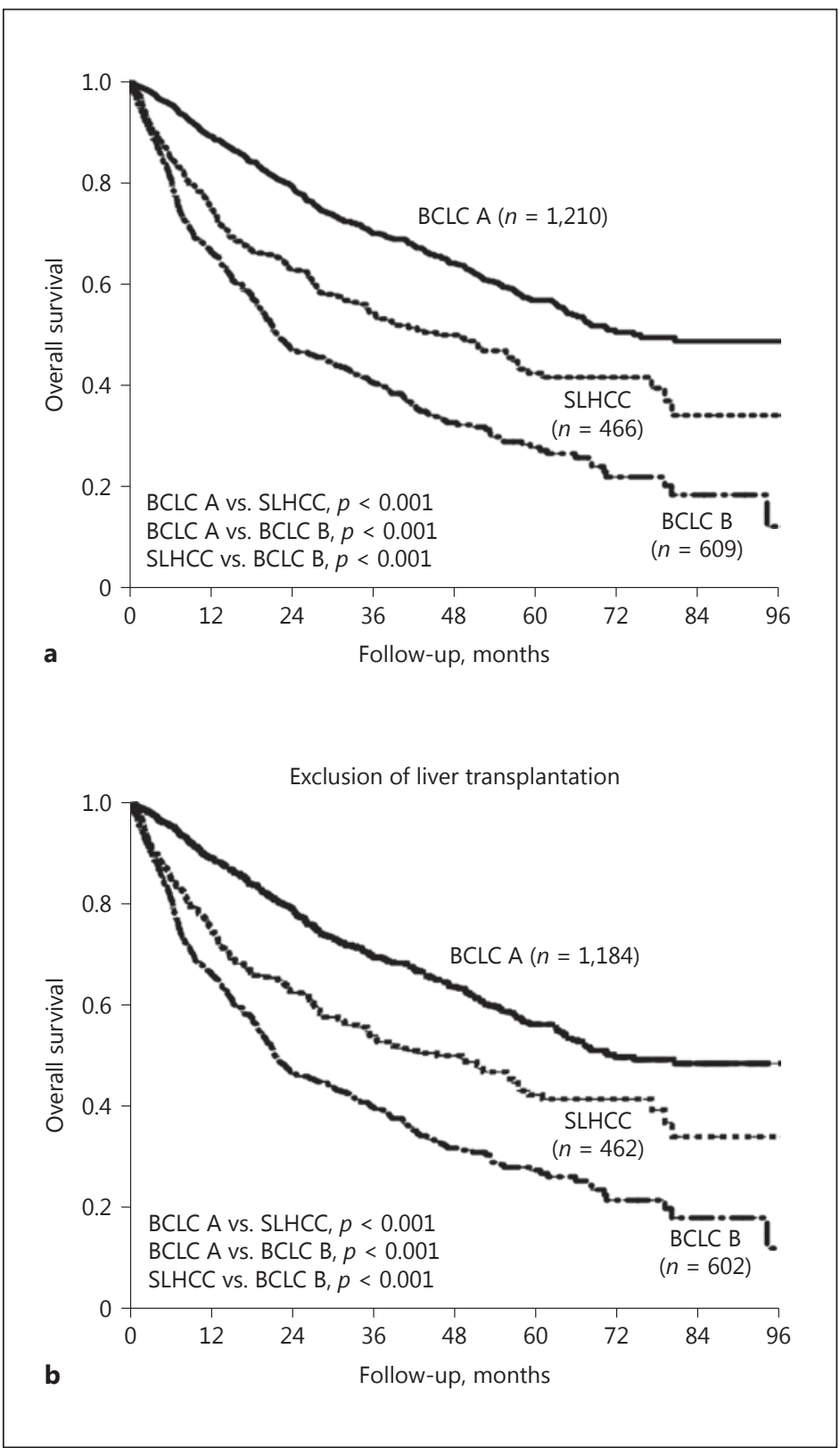

(Figure continued on next pages.)

In model 2, age $>65$ years, serum albumin levels $\leq 3.5 \mathrm{~g} / \mathrm{dL}$, bilirubin levels $>1.0 \mathrm{mg} / \mathrm{dL}$, alkaline phosphatase levels $>100 \mathrm{U} / \mathrm{L}$, creatinine levels $>1.0 \mathrm{mg} / \mathrm{dL}$, AFP levels $>20 \mathrm{ng} / \mathrm{mL}$, noncurative treatment, and HCC group were the risk factors predicting poor OS using multivariate analysis.

\section{Comparison of Patients' OS among the BCLC-A, SLHCC, and BCLC-B Groups Stratified by}

ALBI Grade and Treatment Modality

Since ALBI grade and treatment modalities were crucial in determining OS, we further investigated the impact of these two factors on the prognoses of HCC patients. Stratified by the ALBI grade, we observed significant differences in the OS rates using a pairwise comparison across patients with ALBI grade 1 among the three groups of patients (Fig. 3a). In patients 
Fang et al.: Single Large Hepatocellular Carcinoma

\begin{tabular}{|c|c|c|c|c|c|c|c|c|}
\hline Subgroup & $\begin{array}{l}\text { BCLC-A } \\
\text { patients }\end{array}$ & Deaths & $\begin{array}{l}\text { SLHCC } \\
\text { patients }\end{array}$ & Deaths & Hazard ratio $(95 \% \mathrm{CI})$ & $p$ & & \\
\hline \multicolumn{9}{|l|}{ Age } \\
\hline$\leq 65$ years & 562 & 150 & 195 & 74 & $1.700(1.286-2.247)$ & $<0.001$ & $\longmapsto$ & \\
\hline$>65$ years & 648 & 216 & 271 & 121 & $1.747(1.398-2.184)$ & $<0.001$ & $\longmapsto$ & \\
\hline \multicolumn{9}{|l|}{ Sex } \\
\hline Male & 882 & 261 & 375 & 160 & $1.888(1.550-2.299)$ & $<0.001$ & $\longmapsto$ & \\
\hline Female & 328 & 105 & 91 & 35 & $1.295(0.883-1.900)$ & 0.185 & 11 & \\
\hline \multicolumn{9}{|l|}{$\mathrm{HBsAg}$} \\
\hline Positive & 599 & 173 & 228 & 100 & $1.905(1.489-2.438)$ & $<0.001$ & $\longmapsto$ & \\
\hline Negative & 452 & 139 & 203 & 82 & 1.971 (1.574-2.468) & $<0.001$ & $\longmapsto$ & \\
\hline \multicolumn{9}{|l|}{ Anti-HCV } \\
\hline Positive & 380 & 121 & 84 & 37 & $1.920(1.327-2.777)$ & 0.001 & $\longmapsto$ & \\
\hline Negative & 605 & 165 & 336 & 142 & 1.971 (1.574-2.468) & $<0.001$ & $\longmapsto$ & \\
\hline \multicolumn{9}{|c|}{ Antiviral therapy } \\
\hline Yes & 359 & 106 & 54 & 29 & $2.298(1.524-3.467)$ & $<0.001$ & $\longrightarrow$ & \\
\hline No & 851 & 260 & 412 & 166 & 1.597 (1.314-1.941) & $<0.001$ & $\longmapsto$ & \\
\hline \multicolumn{9}{|c|}{ 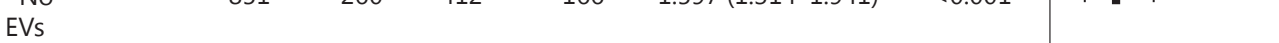 } \\
\hline With EVs & 215 & 114 & 43 & 23 & $1.824(1.162-2.864)$ & 0.009 & $\longmapsto$ & \\
\hline Without EVs & 908 & 207 & 376 & 142 & $1.962(1.584-2.429)$ & $<0.001$ & $\longmapsto$ & \\
\hline \multicolumn{9}{|l|}{ Albumin } \\
\hline$>3.5 \mathrm{~g} / \mathrm{dL}$ & 797 & 202 & 293 & 103 & $1.610(1.270-2.042)$ & $<0.001$ & $\longmapsto$ & \\
\hline$\leq 3.5 \mathrm{~g} / \mathrm{dL}$ & 383 & 152 & 166 & 89 & $1.992(1.532-2.590)$ & $<0.001$ & 1 & \\
\hline \multicolumn{9}{|l|}{ Bilirubin } \\
\hline$>1.0 \mathrm{mg} / \mathrm{dL}$ & 378 & 159 & 135 & 72 & $1.643(1.243-2.172)$ & $<0.001$ & $\longmapsto$ & \\
\hline$\leq 1.0 \mathrm{mg} / \mathrm{dL}$ & 818 & 203 & 323 & 119 & $1.861(1.483-2.334)$ & $<0.001$ & $\longmapsto 1$ & \\
\hline \multicolumn{9}{|l|}{ ALT } \\
\hline$>40 \mathrm{U} / \mathrm{L}$ & 573 & 195 & 245 & 110 & $1.774(1.403-2.242)$ & $<0.001$ & $\longmapsto$ & \\
\hline$\leq 40 \mathrm{U} / \mathrm{L}$ & 629 & 170 & 220 & 85 & $1.672(1.289-2.170)$ & $<0.001$ & $\longmapsto$ & \\
\hline \multicolumn{9}{|l|}{ AST } \\
\hline$>45 \mathrm{U} / \mathrm{L}$ & 511 & 198 & 236 & 120 & $1.829(1.457-2.296)$ & $<0.001$ & $\longmapsto$ & \\
\hline$\leq 45 \mathrm{U} / \mathrm{L}$ & 648 & 155 & 215 & 68 & $1.513(1.138-2.013)$ & 0.004 & $\longmapsto$ & \\
\hline \multicolumn{9}{|c|}{$1.310(1.130-2.010)$} \\
\hline$>100 \mathrm{U} / \mathrm{L}$ & 252 & 103 & 186 & 93 & $1.626(1.227-2.155)$ & 0.001 & $\longmapsto$ & \\
\hline$\leq 100 \mathrm{U} / \mathrm{L}$ & 613 & 173 & 202 & 74 & $1.566(1.192-2.057)$ & 0.001 & $\longmapsto$ & \\
\hline \multicolumn{9}{|l|}{ Creatinine } \\
\hline$>1.0 \mathrm{mg} / \mathrm{dL}$ & 455 & 152 & 189 & 82 & $1.610(1.231-2.108)$ & 0.001 & $\longmapsto$ & \\
\hline$\leq 1.0 \mathrm{mg} / \mathrm{dL}$ & 750 & 214 & 274 & 112 & $1.797(1.429-2.259)$ & $<0.001$ & $\longmapsto 1$ & \\
\hline \multicolumn{9}{|l|}{ PT/INR } \\
\hline$>1.1$ & 414 & 163 & 118 & 65 & $1.983(1.486-2.646)$ & $<0.001$ & $\longmapsto$ & \\
\hline$\leq 1.1$ & 782 & 202 & 344 & 128 & $1.794(1.437-2.240)$ & $<0.001$ & $\longmapsto$ & \\
\hline \multicolumn{9}{|l|}{ AFP } \\
\hline$>20 \mathrm{ng} / \mathrm{mL}$ & 558 & 223 & 260 & 129 & $1.694(1.363-2.105)$ & $<0.001$ & $\longmapsto \mid$ & \\
\hline$\leq 20 \mathrm{ng} / \mathrm{mL}$ & 628 & 137 & 198 & 62 & $1.593(1.180-2.151)$ & 0.002 & $\longmapsto$ & \\
\hline \multicolumn{9}{|l|}{ Tumor number } \\
\hline Single & 942 & 266 & 466 & 195 & $1.886(1.567-2.269)$ & $<0.001$ & $\longmapsto$ & \\
\hline Multiple & 268 & 100 & 0 & 0 & & & & \\
\hline c & & & & & & & 2 & 3 \\
\hline
\end{tabular}

with ALBI grade 2 or 3, the patients in the BCLC-A group had significantly higher OS rates than those in the SLHCC or BCLC-B groups (Fig. 3b, c). However, the OS rates were comparable between the SLHCC group and the BCLC-B group.

When stratified by treatment modalities, patients who underwent curative treatment in the BCLC-B group had a significantly lower OS rate than those in the BCLC-A or SLHCC groups (Fig. 3d). The patients in the SLHCC group had a trend toward lower OS rates than those in the BCLC-A group, but this was not statistically significant $(p=0.055)$. For patients who underwent TACE or other treatment modalities, the BCLC-A group had a significantly higher OS rate than the SLHCC group or the BCLC-B group (Fig. 3e, f). However, the OS rates were similar between the SLHCC group and the BCLC-B group. 


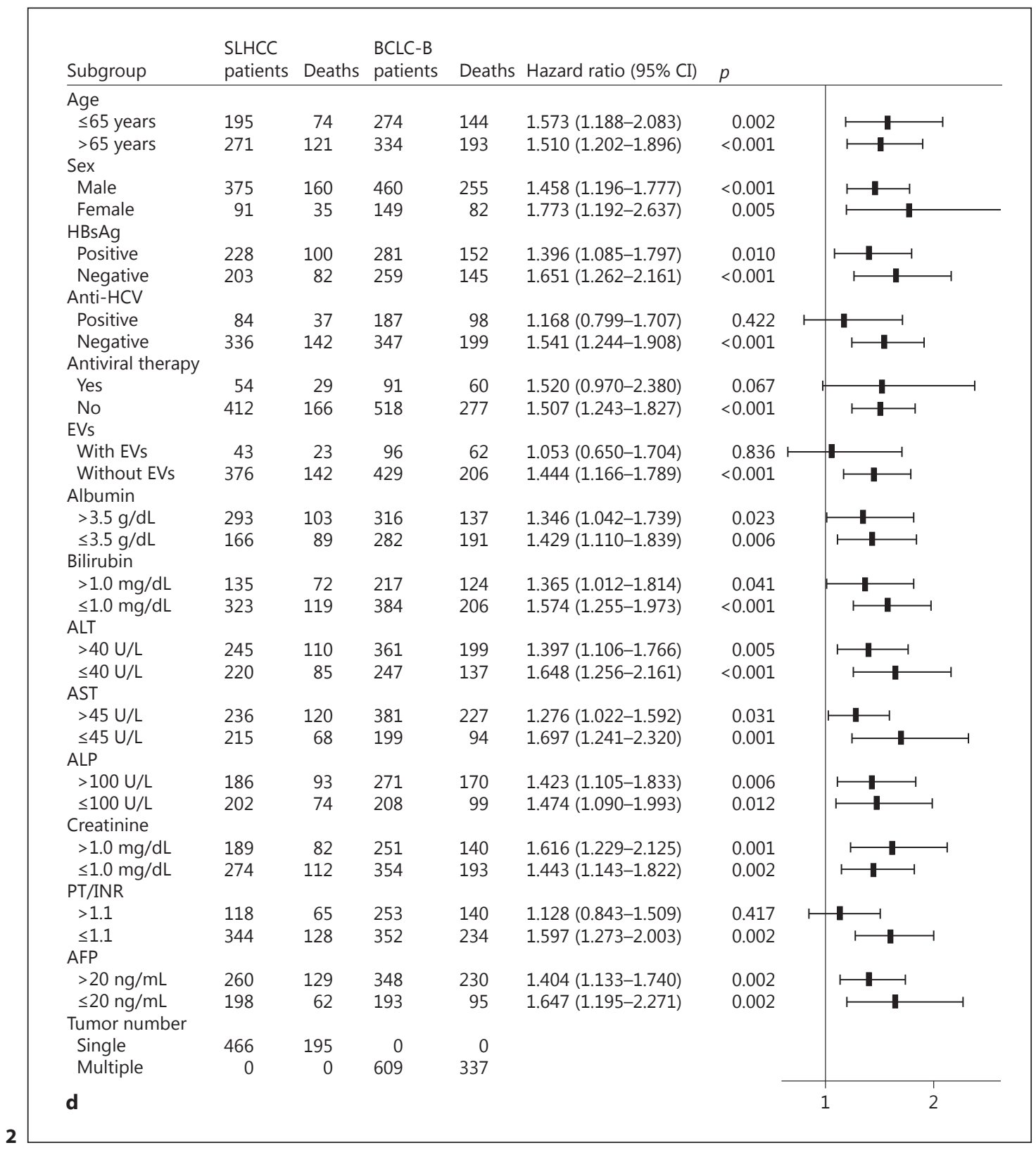

Interaction between ALBI Grade and Treatment Modalities in Determining HCC Prognosis

For patients with an ALBI grade 1 who underwent curative treatment, the OS rates were similar between the BCLC-A and the SLHCC group. Also, both groups has significantly higher OS rates than the BCLC-B group (Fig. 4a). However, for those with an ALBI grade 1 who underwent noncurative treatment, the OS rates were comparable across the three groups (Fig. 4b).

Regarding patients with ALBI grade 2 or 3, patients in the BCLC-A group had a significantly higher OS rate than those in the BCLC-B group, irrespective of treatment modalities (Fig. 4c, d). Nevertheless, in the setting of curative treatment, patients in the BCLC-A group had a trend toward higher OS rates than those in the SLHCC group, although this was not statistically significant $(p=0.058)$. Regarding patients who underwent noncurative treatment, 
Fang et al.: Single Large Hepatocellular Carcinoma

\begin{tabular}{|c|c|c|c|c|c|c|c|}
\hline Subgroup & $\begin{array}{l}\text { BCLC-A } \\
\text { patients }\end{array}$ & Deaths & $\begin{array}{l}\text { BCLC-B } \\
\text { patients }\end{array}$ & Deaths & Hazard ratio $(95 \% \mathrm{CI})$ & $p$ & \\
\hline \multicolumn{8}{|l|}{ Age } \\
\hline$\leq 65$ years & 562 & 150 & 274 & 144 & 1.651 (1.472-1.852) & $<0.001$ & $\mapsto$ \\
\hline$>65$ years & 648 & 216 & 334 & 193 & 1.651 (1.497-1.820) & $<0.001$ & $\mapsto-1$ \\
\hline \multicolumn{8}{|l|}{ Sex } \\
\hline Male & 882 & 261 & 460 & 255 & $1.678(1.539-1.831)$ & $<0.001$ & $\mapsto-1$ \\
\hline Female & 328 & 105 & 149 & 82 & 1.556 (1.346-1.799) & $<0.001$ & $\longmapsto$ \\
\hline \multicolumn{8}{|l|}{$\mathrm{HBsAg}$} \\
\hline Positive & 599 & 173 & 281 & 152 & 1.653 (1.482-1.844) & $<0.001$ & $\mapsto$ \\
\hline Negative & 452 & 139 & 259 & 145 & $1.643(1.464-1.845)$ & $<0.001$ & $\mapsto-1$ \\
\hline \multicolumn{8}{|l|}{ Anti-HCV } \\
\hline Positive & 380 & 121 & 187 & 98 & 1.509 (1.320-1.725) & $<0.001$ & $\mapsto$ \\
\hline Negative & 605 & 165 & 347 & 199 & $1.772(1.598-1.965)$ & $<0.001$ & $\mapsto$ \\
\hline \multicolumn{8}{|c|}{ Antiviral therapy } \\
\hline Yes & 359 & 106 & 91 & 60 & $1.914(1.629-2.249)$ & $<0.001$ & $\longmapsto$ \\
\hline No & 851 & 260 & 518 & 277 & $1.570(1.442-1.709)$ & $<0.001$ & $\mapsto-1$ \\
\hline \multicolumn{8}{|c|}{ 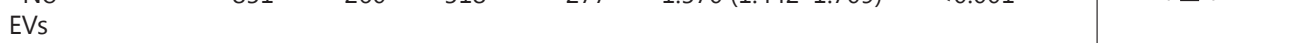 } \\
\hline With EVs & 215 & 114 & 96 & 62 & 1.390 (1.189-1.625) & $<0.001$ & $\mapsto$ \\
\hline Without EVs & 908 & 207 & 429 & 206 & $1.698(1.542-1.871)$ & $<0.001$ & $\mapsto-1$ \\
\hline \multicolumn{8}{|l|}{ Albumin } \\
\hline$>3.5 \mathrm{~g} / \mathrm{dL}$ & 797 & 202 & 316 & 137 & 1.714 (1.539-1.910) & $<0.001$ & $\mapsto-1$ \\
\hline$\leq 3.5 \mathrm{~g} / \mathrm{dL}$ & 383 & 152 & 282 & 191 & $1.483(1.330-1.654)$ & $<0.001$ & $\mapsto-1$ \\
\hline \multicolumn{8}{|l|}{ Bilirubin } \\
\hline$>1.0 \mathrm{mg} / \mathrm{dL}$ & 378 & 159 & 217 & 124 & $1.515(1.345-1.706)$ & $<0.001$ & $\mapsto-1$ \\
\hline$\leq 1.0 \mathrm{mg} / \mathrm{dL}$ & 818 & 203 & 384 & 206 & 1.734 (1.573-1.912) & $<0.001$ & $\mapsto$ \\
\hline \multicolumn{8}{|c|}{$1 . / 34(1.3 / 3-1.912)$} \\
\hline$>40 \mathrm{U} / \mathrm{L}$ & 573 & 195 & 361 & 199 & $1.606(1.453-1.774)$ & $<0.001$ & $\mapsto-1$ \\
\hline$\leq 40 \mathrm{U} / \mathrm{L}$ & 629 & 170 & 247 & 137 & $1.684(1.504-1.886)$ & $<0.001$ & $\mapsto-1$ \\
\hline \multicolumn{8}{|l|}{ AST } \\
\hline$>45 \mathrm{U} / \mathrm{L}$ & 511 & 198 & 381 & 227 & 1.551 (1.409-1.708) & $<0.001$ & $H=-1$ \\
\hline$\leq 45 \mathrm{U} / \mathrm{L}$ & 648 & 155 & 199 & 94 & $1.614(1.419-1.835)$ & $<0.001$ & $\mapsto$ \\
\hline \multicolumn{8}{|c|}{$1.014(1.419-1.030)$} \\
\hline$>100 \mathrm{U} / \mathrm{L}$ & 252 & 103 & 271 & 170 & $1.534(1.356-1.736)$ & $<0.001$ & $\mapsto$ \\
\hline$\leq 100 \mathrm{U} / \mathrm{L}$ & 613 & 173 & 208 & 99 & $1.533(1.354-1.736)$ & $<0.001$ & $\mapsto$ \\
\hline \multicolumn{8}{|l|}{ Creatinine } \\
\hline$>1.0 \mathrm{mg} / \mathrm{dL}$ & 455 & 152 & 251 & 140 & 1.646 (1.466-1.849) & $<0.001$ & $\mapsto$ \\
\hline$\leq 1.0 \mathrm{mg} / \mathrm{dL}$ & 750 & 214 & 354 & 193 & $1.633(1.481-1.800)$ & $<0.001$ & $1-1$ \\
\hline \multicolumn{8}{|l|}{ PT/INR } \\
\hline$>1.1$ & 414 & 163 & 253 & 140 & 1.500 (1.343-1.675) & $<0.001$ & $\mapsto-1$ \\
\hline \multirow{2}{*}{\multicolumn{8}{|c|}{$1.11 /(1.352-1.900)$}} \\
\hline & & & & & & & \\
\hline$>20 \mathrm{ng} / \mathrm{mL}$ & 558 & 223 & 348 & 230 & $1.557(1.420-1.708)$ & $<0.001$ & $\mapsto-1$ \\
\hline$\leq 20 \mathrm{ng} / \mathrm{mL}$ & 628 & 137 & 193 & 95 & $1.643(1.441-1.875)$ & $<0.001$ & $\mapsto$ \\
\hline \multicolumn{8}{|l|}{ Tumor number } \\
\hline Single & 942 & 266 & 0 & 0 & & & \\
\hline Multiple & 268 & 100 & 609 & 337 & $1.422(1.271-1.590)$ & $<0.001$ & $\mapsto-1$ \\
\hline e & & & & & & & 2 \\
\hline
\end{tabular}

patients in the BCLC-A group had a significantly higher OS rate than those in the SLHCC group $(p<0.001)$. The OS rates were comparable between the SLHCC and the BCLC-B group, both in terms of curative and noncurative treatment.

\section{Prognoses of Patients in the SLHCC Group}

Subsequently, we analyzed the factors associated with poor prognosis in patients with SLHCC. When stratified by tumor size, patients with a tumor sized between 5 and $10 \mathrm{~cm}$ had a significantly higher OS rate than those with a tumor size $>10 \mathrm{~cm}$, except for patients who underwent noncurative treatment modalities (Fig. 5). Moreover, patients who underwent curative treatment had a better OS than those who underwent noncurative treatment, irrespective of tumor size and ALBI grade (Fig. 6). 
Fang et al.: Single Large Hepatocellular Carcinoma

Table 2. Univariate and multivariate analysis of factors associated with poor OS in model 1

\begin{tabular}{|c|c|c|c|c|c|}
\hline \multirow[t]{2}{*}{ Variable } & \multirow[t]{2}{*}{ Cases, $n$} & \multicolumn{2}{|l|}{ Univariate analysis } & \multicolumn{2}{|l|}{ Multivariate analysis } \\
\hline & & $\operatorname{HR}(95 \% \mathrm{CI})$ & $p$ & $\operatorname{HR}(95 \% \mathrm{CI})$ & $p$ \\
\hline Age $(>65 / \leq 65$ years $)$ & $1,254 / 1,031$ & $1.367(1.197-1.562)$ & $<0.001$ & $1.230(1.048-1.443)$ & 0.011 \\
\hline Sex (female/male) & $568 / 1,717$ & $1.001(0.860-1.165)$ & 0.993 & & \\
\hline HBsAg (+/-) & $1,111 / 927$ & $0.870(0.757-0.999)$ & 0.049 & & \\
\hline Anti-HCV (+/-) & $652 / 1,305$ & 0.997 (0.858-1.159) & 0.974 & & \\
\hline Antiviral therapy (no/yes) & $1,781 / 504$ & $1.249(1.065-1.464)$ & 0.006 & & \\
\hline EVs (with/without) & $354 / 1,713$ & $1.805(1.531-2.123)$ & $<0.001$ & & \\
\hline Albumin $(\leq 3.5 />3.5 \mathrm{~g} / \mathrm{dL})$ & $831 / 1,406$ & $2.358(2.062-2.695)$ & $<0.001$ & & \\
\hline Bilirubin $(>1.0 / \leq 1.0 \mathrm{mg} / \mathrm{dL})$ & $730 / 1,525$ & $1.758(1.536-2.011)$ & $<0.001$ & & \\
\hline $\operatorname{ALT}(>40 / \leq 40 \mathrm{U} / \mathrm{L})$ & $1,179 / 1,096$ & $1.150(1.007-1.312)$ & 0.038 & & \\
\hline $\operatorname{AST}(>45 / \leq 45 \mathrm{U} / \mathrm{L})$ & $1,128 / 1,062$ & $1.962(1.708-2.254)$ & $<0.001$ & & \\
\hline $\operatorname{ALP}(>100 / \leq 100 \mathrm{U} / \mathrm{L})$ & $709 / 1,023$ & $2.124(1.833-2.462)$ & $<0.001$ & $1.449(1.234-1.703)$ & $<0.001$ \\
\hline Creatinine $(>1.0 / \leq 1.0 \mathrm{mg} / \mathrm{dL})$ & $895 / 1,378$ & $1.274(1.1716-1.456)$ & $<0.001$ & 1.417 (1.212-1.658) & $<0.001$ \\
\hline $\mathrm{PT} / \mathrm{INR}(>1.1 / \leq 1.1)$ & $785 / 1,478$ & $1.729(1.513-1.975)$ & $<0.001$ & & \\
\hline Platelets $\left(\leq 10^{5} />10^{5} / \mathrm{mm}^{3}\right)$ & $136 / 2,140$ & $1.099(0.836-1.443)$ & 0.501 & & \\
\hline Multiple tumors (yes/no) & $877 / 1,408$ & $1.886(1.654-2.151)$ & $<0.001$ & & \\
\hline $\operatorname{AFP}(>20 / \leq 20 \mathrm{ng} / \mathrm{mL})$ & $1,202 / 1,040$ & $2.063(1.794-2.374)$ & $<0.001$ & $1.850(1.574-2.174)$ & $<0.001$ \\
\hline Noncurative/curative treatment & $928 / 1,357$ & $3.422(2.991-3.914)$ & $<0.001$ & $2.429(2.047-2.884)$ & $<0.001$ \\
\hline ALBI grade 1 & 933 & 1 & & 1 & \\
\hline ALBI grade 2 & 1,143 & $2.201(1.892-2.559)$ & $<0.001$ & $1.659(1.389-1.981)$ & $<0.001$ \\
\hline ALBI grade 3 & 209 & $4.385(3.541-5.430)$ & $<0.001$ & $3.233(2.506-4.171)$ & $<0.001$ \\
\hline BCLC-A group & 1,210 & 1 & & 1 & \\
\hline SLHCC group & 466 & $1.726(1.451-2.054)$ & $<0.001$ & $1.453(1.190-1.776)$ & $<0.001$ \\
\hline BCLC-B group & 609 & $2.671(2.302-3.099)$ & $<0.001$ & $1.493(1.235-1.804)$ & $<0.001$ \\
\hline
\end{tabular}

In model 1, the ALBI grade was enrolled, but albumin and bilirubin levels were not entered into the multivariate analysis. In model 2 , we selected albumin and bilirubin, but the ALBI grade was not enrolled in the multivariate analysis. Age $>65$ years (HR $1.214,95 \%$ CI 1.038-1.419, $p=0.015$ ), serum albumin levels $\leq 3.5 \mathrm{~g} / \mathrm{dL}$ (HR 1.736, 95\% CI 1.477-2.041, $p<0.001$ ), bilirubin $>1.0 \mathrm{mg} / \mathrm{dL}$ (HR 1.338 , 95\% CI 1.135-1.577, $p=0.001$ ), ALP >100 U/L (HR 1.478, 95\% CI 1.255-1.741, $p<0.001$ ), creatinine >1.0 mg/dL (HR 1.461, 95\% CI $1.245-1.714, p<0.001$ ), AFP $>20 \mathrm{ng} / \mathrm{mL}$ (HR 1.887, 95\% CI 1.603-2.222, $p<0.001$ ), noncurative therapy (HR 2.420, 95\% CI 2.035$2.877, p<0.001$ ), and HCC group (SLHCC group, HR 1.384, 95\% CI 1.131-1.694, $p=0.002$; BCLC-B group, HR 1.497, 95\% CI 1.235-1.814, $p<0.001$ ) were the independent risk factors associated with poor OS. AFP, alpha-fetoprotein; ALBI, albumin-bilirubin; ALP, alkaline phosphatase; ALT, alanine aminotransferase; AST, aspartate aminotransferase; BCLC, Barcelona Clinic Liver Cancer; CI, confidence interval; EVs, esophageal varices; HBsAg, hepatitis B virus surface antigen; HCC, hepatocellular carcinoma; HCV, hepatitis C virus; HR, hazard ratio; OS, overall survival; PT/INR, prothrombin time/international normalized ratio.

As shown in Table 3, multivariate analysis disclosed that serum AFP $>20 \mathrm{mg} / \mathrm{mL}$, tumor size $>10 \mathrm{~cm}$, noncurative treatment, and higher ALBI grades were the independent risk factors associated with poor OS.

\section{Comparison of OS between Resection Surgery and TACE in the SLHCC Group}

We further compared the prognoses between resection surgery and TACE in the SLHCC group. Patients who underwent resection surgery had significantly higher OS rates than those who received TACE ( $p<0.001$; Fig. 7a), especially in the setting of tumor size between 5 and $10 \mathrm{~cm}(p<0.001$; Fig. $7 \mathrm{~b})$. For patients with a tumor size $>10 \mathrm{~cm}$, resection surgery had a trend toward a higher OS rate than TACE ( $p=0.055$; Fig. 7c).

When stratified by liver functional reserve, resection surgery provided a significant survival benefit compared with TACE both in patients with ALBI grade 1 ( $p=0.009$; Fig. $7 \mathrm{~d}$ ) and in those with ALBI grade 2 or 3 ( $p<0.001$; Fig. 7e). 

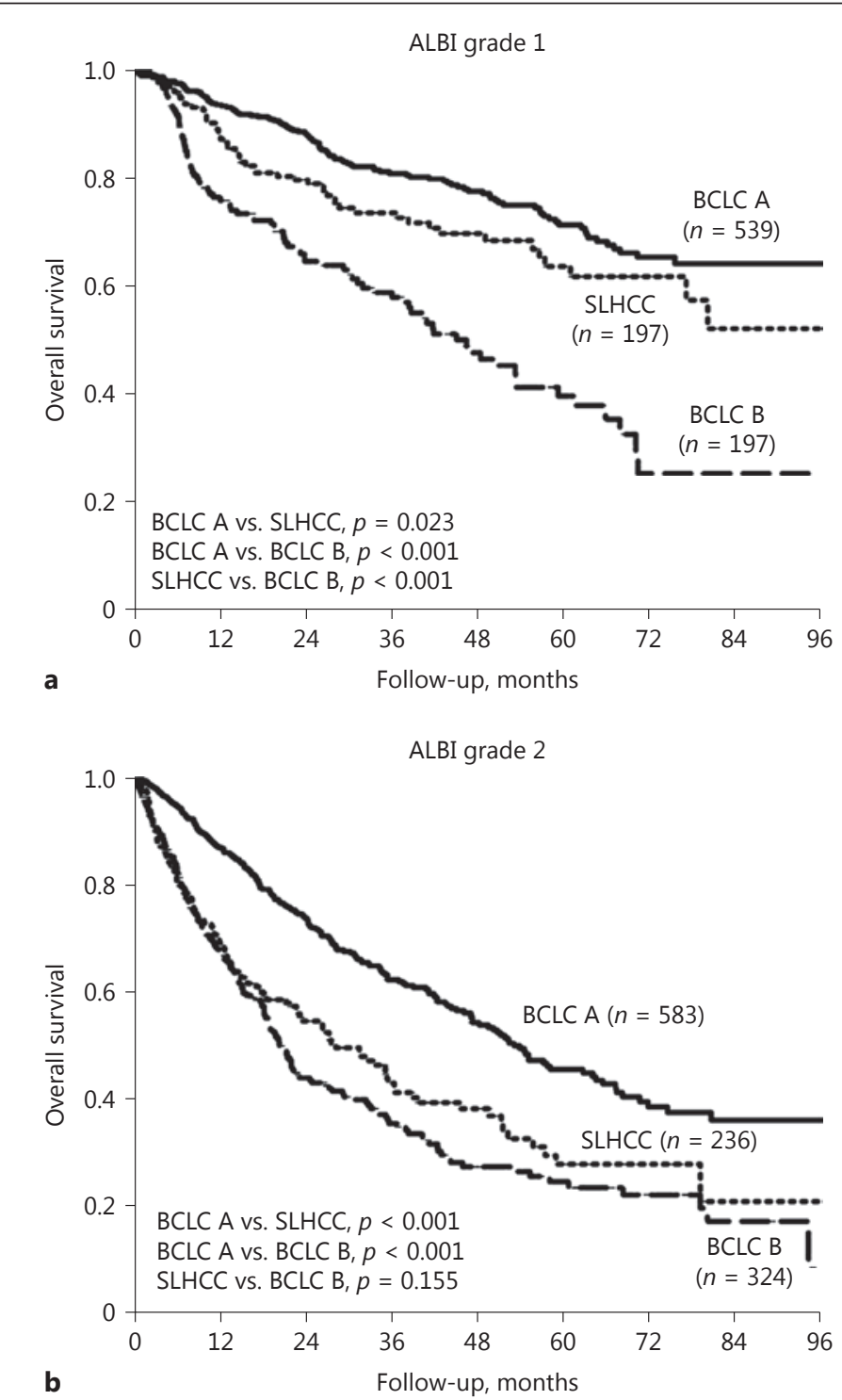

Fig. 3. Comparison of $\mathrm{OS}$ rates among the BCLC-A, SLHCC, and BCLC-B groups stratified by ALBI grade and treatment modality. a ALBI grade 1 . b ALBI grade 2 . c ALBI grade 3. d Curative treatment. e TACE. $\mathbf{f}$ Other treatments. ALBI, albumin-bilirubin; BCLC, Barcelona Clinic Liver Cancer; OS, overall survival; SLHCC, single large hepatocellular carcinoma; TACE, transarterial chemoembolization.

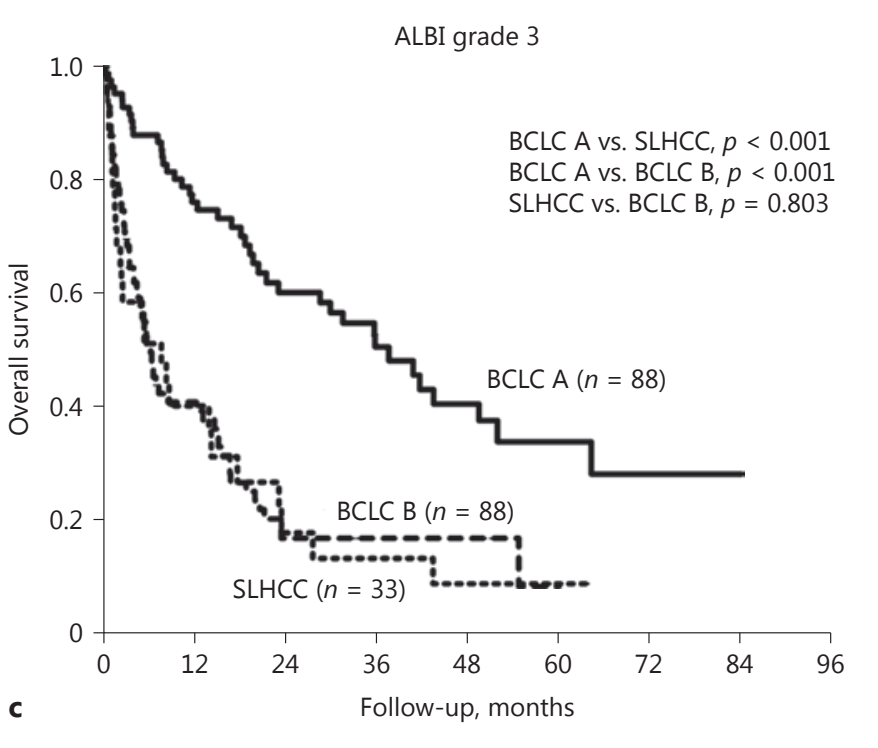




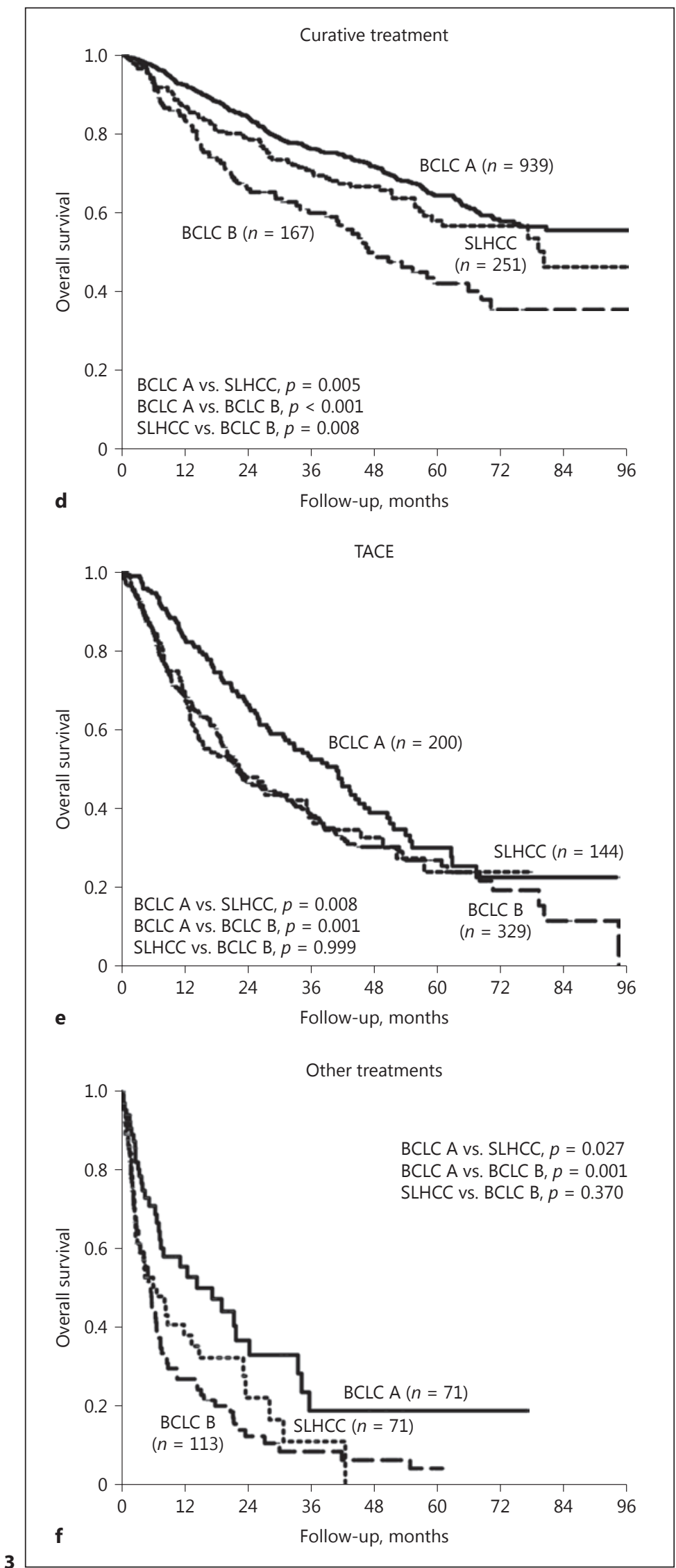


Fang et al.: Single Large Hepatocellular Carcinoma

Fig. 4. Interaction between ALBI grade and treatment modalities in determining HCC prognosis. a ALBI grade 1 and curative treatment. b ALBI grade 1 and noncurative treatment. c ALBI grade 2 or 3 and curative treatment. d ALBI grade 2 or 3 and noncurative treatment. ALBI, albumin-bilirubin; BCLC, Barcelona Clinic Liver Cancer; HCC, hepatocellular carcinoma; SLHCC, single large hepatocellular carcinoma.

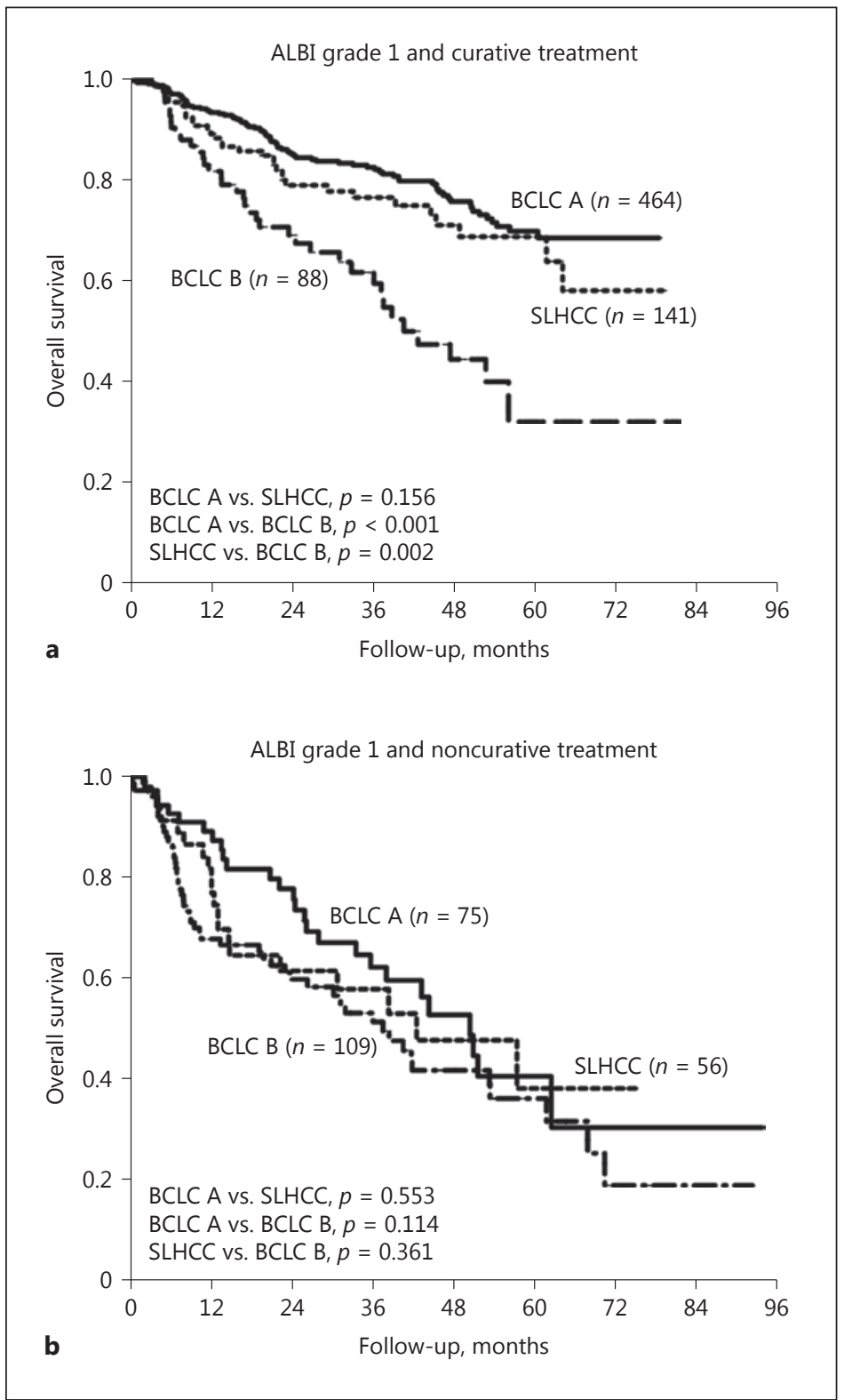

(Figure continued on next page.)

\section{Discussion}

There are several major findings of this study. First, we analyzed a large cohort of patients with SLHCC to comprehensively investigate the impact of tumor size on HCC prognosis. It was demonstrated that patients with SLHCC had an intermediate OS rate to those in the A and B stages of BCLC. Second, the SLHCC group was a heterogeneous group that presented diverse clinical manifestations, treatment modalities, and outcomes. Our study further disclosed that ALBI grade and treatment modalities could help to stratify patients with SLHCC into different prognostic groups. Third, SLHCC patients who underwent curative treatment had a slightly lower OS rate than those in the BCLC-A group. Also, both had significantly better prognosis than patients in the BCLC-B group, especially in the setting of ALBI grade 1 (Fig. 3, 4). Fourth, 
Fang et al.: Single Large Hepatocellular Carcinoma

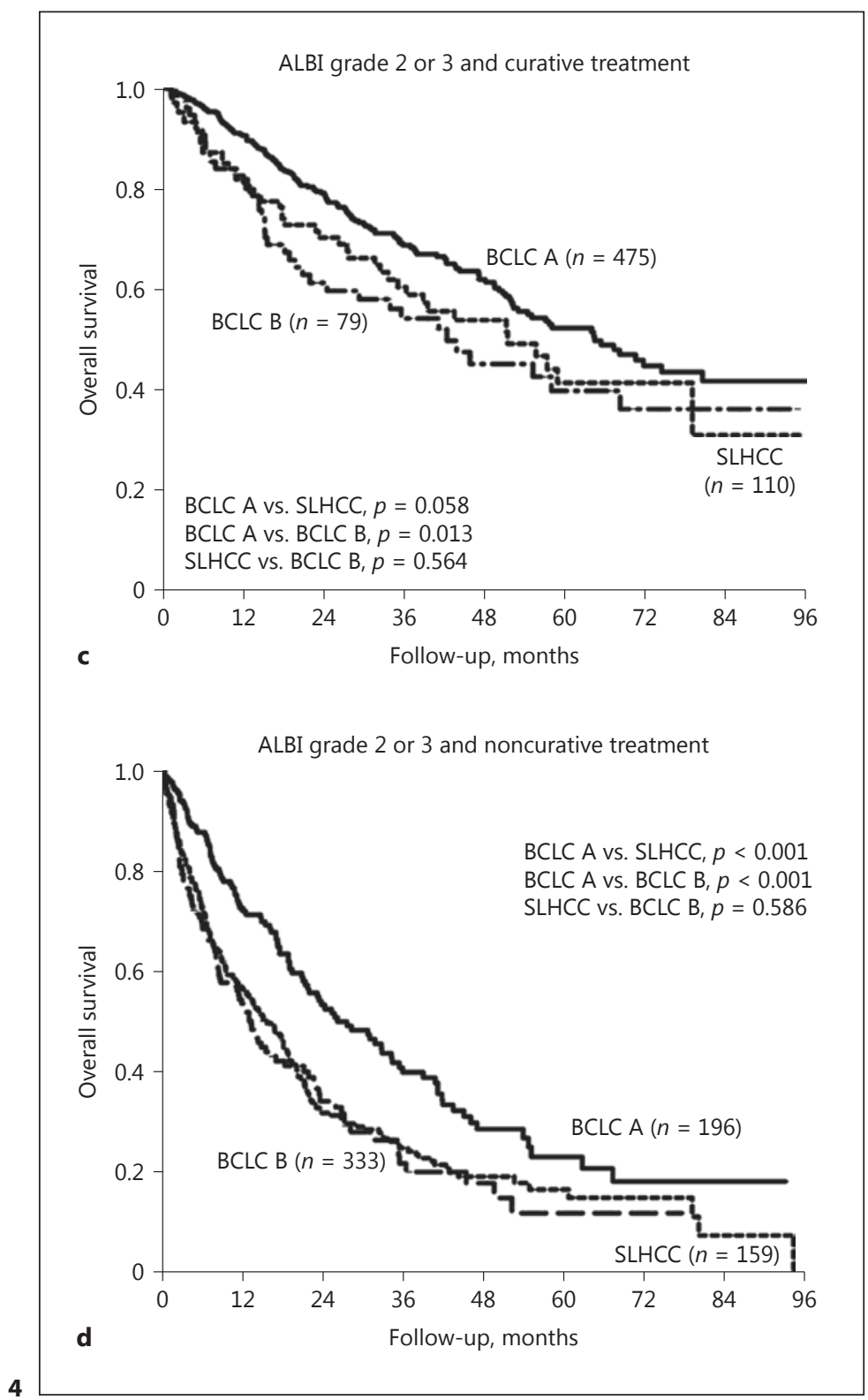

for patients with SLHCC, curative treatment could provide a higher OS rate than noncurative treatment, irrespective of the tumor size and the degree of liver functional reserve. These results indicated that curative treatment was recommended as a first-line therapy for patients with SLHCC if they were not contraindicated.

In our cohort, the 5-year OS rate of patients with SLHCC was $42.6 \%$. This was consistent with the findings of previous studies, which reported patients with SLHCC to have lower 5-year OS rates (36.8-42.9\%) than those with BCLC stage A HCC (55.4-58.6\%) [16, 17]. Our study further demonstrated that SLHCC patients had an intermediate OS rate between those in the BCLC stages A and B. These findings were confirmed by multivariate analysis and most of the subgroup analyses. Therefore, categorizing SLHCC as a distinct and independent group from BCLC stage A and B may be sensible in the future. 
Fig. 5. Comparison of OS rates between patients with a tumor size between 5 and $10 \mathrm{~cm}$ and those with a tumor size $>10 \mathrm{~cm}$ in the SLHCC group stratified by treatment modalities. a All patients $(p=0.001)$. b Patients with curative treatment $(p=0.012)$. c Patients with noncurative treatment ( $p=0.127)$. OS, overall survival; SLHCC, single large hepatocellular carcinoma.
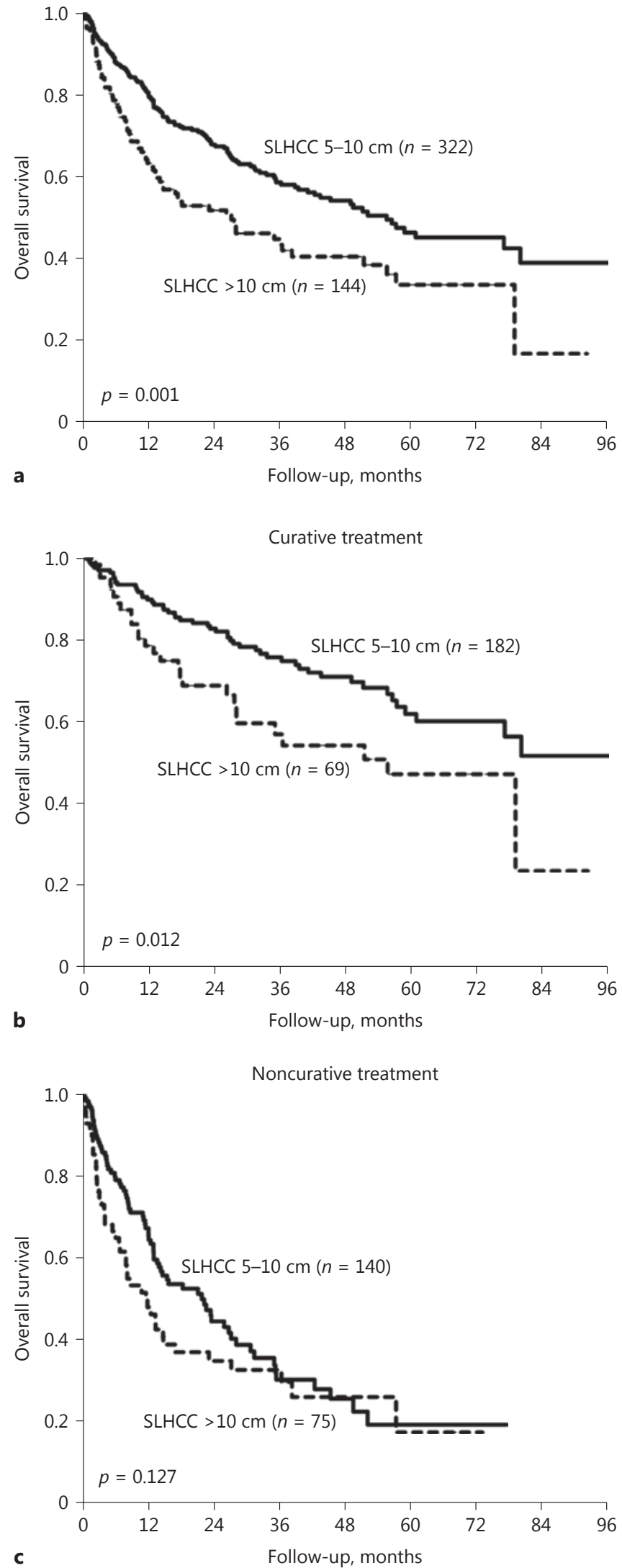


\section{Liver \\ Cancer}

Fig. 6. Comparison of OS rates between patients with curative treatment and those with noncurative treatment in the SLHCC group stratified by tumor size and ALBI grade. a All patients $(p<$ 0.001). b Tumor size between 5 and $10 \mathrm{~cm}(p<0.001)$. c Tumor size $>10 \mathrm{~cm}(p<0.001)$. d ALBI grade $1(p=0.001)$. e ALBI grade 2 or $3(p<0.001)$. ALBI, albuminbilirubin; OS, overall survival; SLHCC, single large hepatocellular carcinoma.
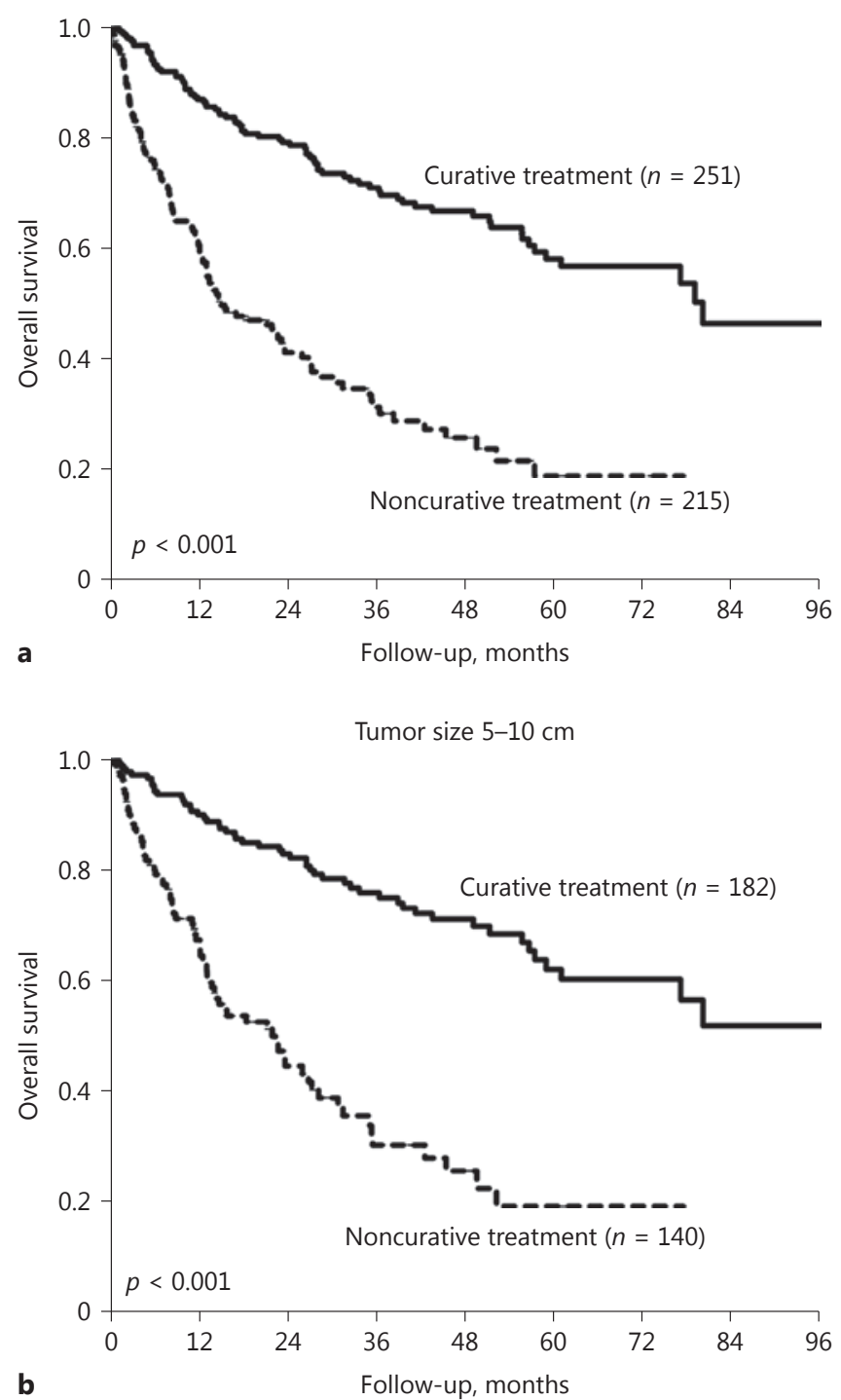

b

Tumor size $>10 \mathrm{~cm}$

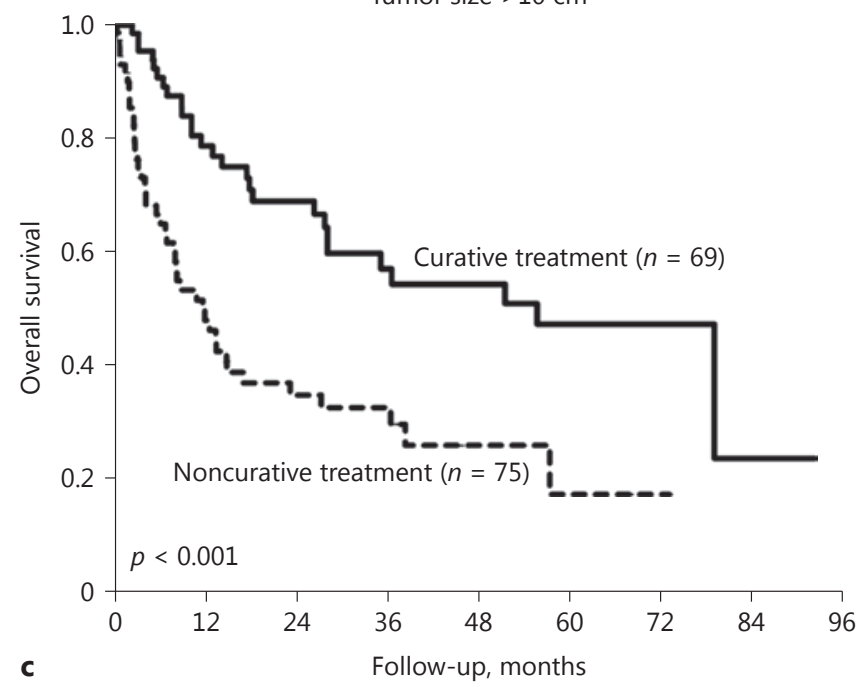

(Figure continued on next page.) 


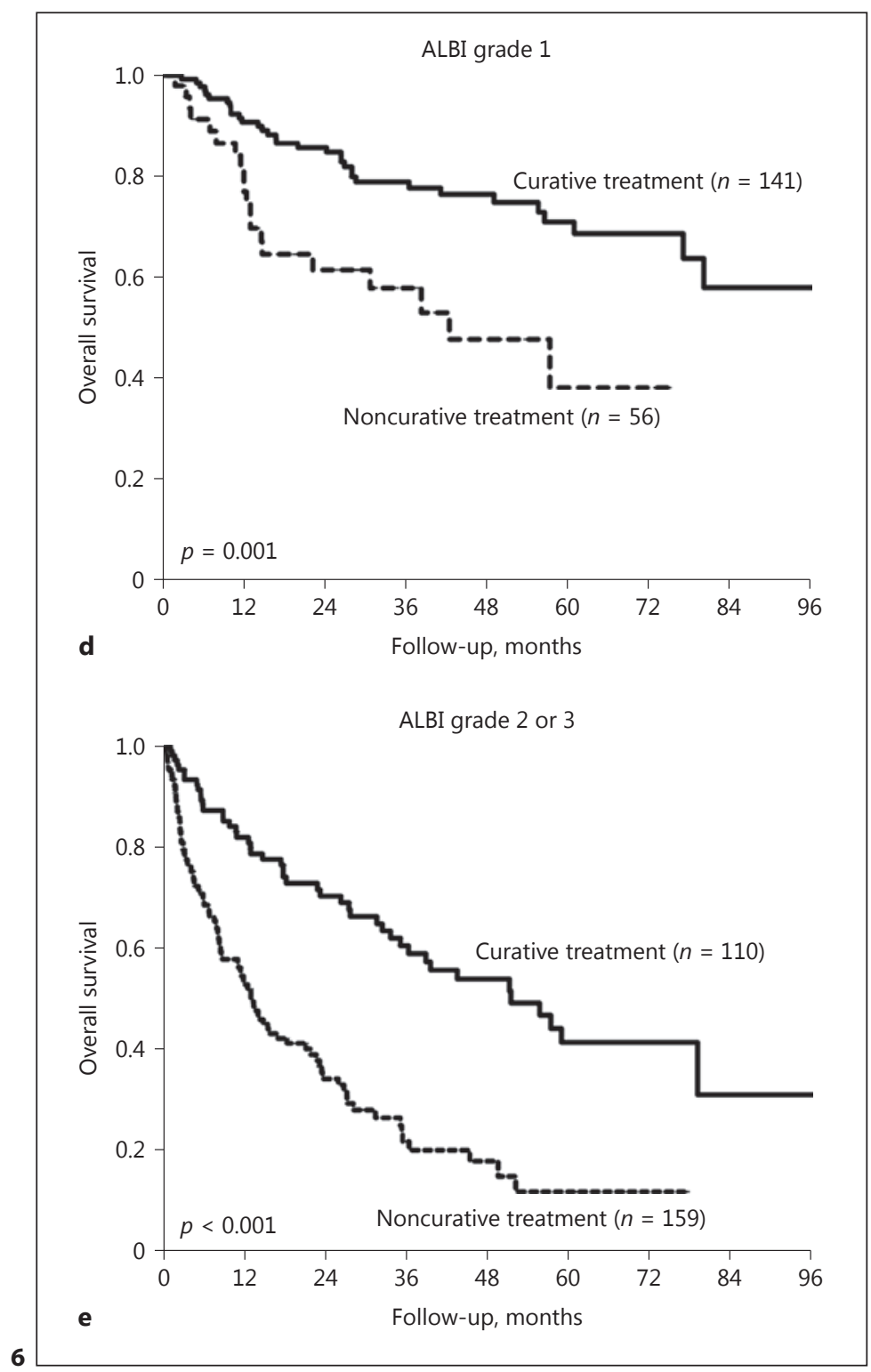

We also showed that age, ALBI grade, serum AFP levels, treatment modalities, and HCC group were important predictors of outcomes for HCC patients. This was consistent with the results from previous studies in which patient factors (such as age and performance status) $[24,25]$, tumor factors (size and number of tumors, vascular invasion, serum AFP level) [26, 27], field factors in the background liver (grade of hepatic inflammation, degree of steatosis, stage of fibrosis, portal hypertension, viral replication) [28-33], and treatment modalities determined the outcomes of HCC patients [9, 34, 35].

Microvascular invasion (MVI) is an important determinant for HCC recurrence and prognosis $[26,36]$. However, MVI could not be diagnosed before liver resection or transplantation. It is critical to search for a surrogate for MVI at the time of HCC diagnosis. Previous studies reported that an increase in tumor size was closely associated with a higher MVI rate in HCC patients [13]. Consequently, in 1996, Mazzaferro et al. [37] proposed the Milan criteria (single tumor $\leq 5 \mathrm{~cm}$ or up to three nodules each $\leq 3 \mathrm{~cm}$ in size and without major vascular invasion) 
Fang et al.: Single Large Hepatocellular Carcinoma

Table 3. Univariate and multivariate analysis of factors associated with poor overall survival for SLHCC group

\begin{tabular}{|c|c|c|c|c|c|}
\hline \multirow[t]{2}{*}{ Variable } & \multirow[t]{2}{*}{ Cases, $n$} & \multicolumn{2}{|l|}{ Univariate analysis } & \multicolumn{2}{|l|}{ Multivariate analysis } \\
\hline & & $\operatorname{HR}(95 \% \mathrm{CI})$ & $p$ & $\operatorname{HR}(95 \% \mathrm{CI})$ & $p$ \\
\hline Age $(>65 / \leq 65$ years $)$ & $271 / 195$ & $1.379(1.032-1.842)$ & 0.030 & & \\
\hline Sex (female/male) & $91 / 375$ & $0.807(0.560-1.164)$ & 0.252 & & \\
\hline $\operatorname{HBsAg}(+/-)$ & $228 / 203$ & $1.043(0.778-1.397)$ & 0.782 & & \\
\hline Anti-HCV (+/-) & $84 / 336$ & $1.155(0.803-1.658)$ & 0.438 & & \\
\hline Antiviral therapy (no/yes) & $412 / 54$ & $0.845(0.569-1.255)$ & 0.405 & & \\
\hline EVs (with/without) & $43 / 376$ & $2.114(1.359-3.289)$ & 0.001 & & \\
\hline Albumin $(\leq 3.5 />3.5 \mathrm{~g} / \mathrm{dL})$ & $166 / 293$ & $2.309(1.736-3.077)$ & $<0.001$ & & \\
\hline Bilirubin $(>1.0 / \leq 1.0 \mathrm{mg} / \mathrm{dL})$ & $135 / 323$ & $1.756(1.310-2.355)$ & $<0.001$ & & \\
\hline $\operatorname{ALT}(>40 / \leq 40 \mathrm{U} / \mathrm{L})$ & $245 / 220$ & $1.185(0.892-1.573)$ & 0.241 & & \\
\hline AST $(>45 / \leq 45 \mathrm{U} / \mathrm{L})$ & $236 / 215$ & $2.067(1.534-2.786)$ & $<0.001$ & & \\
\hline $\operatorname{ALP}(>100 / \leq 100 \mathrm{U} / \mathrm{L})$ & $186 / 202$ & $1.870(1.376-2.542)$ & $<0.001$ & & \\
\hline Creatinine $(>1.0 / \leq 1.0 \mathrm{mg} / \mathrm{dL})$ & $189 / 274$ & $1.139(0.856-1.515)$ & 0.371 & & \\
\hline $\mathrm{PT} / \mathrm{INR}(>1.1 / \leq 1.1)$ & $118 / 344$ & $1.959(1.450-2.646)$ & $<0.001$ & & \\
\hline Platelets $\left(\leq 10^{5} />10^{5} / \mathrm{mm}^{3}\right)$ & $10 / 455$ & $1.439(0.591-3.497)$ & 0.423 & & \\
\hline $\operatorname{AFP}(>20 / \leq 20 \mathrm{ng} / \mathrm{mL})$ & $260 / 198$ & $2.003(1.478-2.713)$ & $<0.001$ & $2.223(1.530-3.231)$ & $<0.001$ \\
\hline Tumor size $(>10 / \leq 10 \mathrm{~cm})$ & $144 / 322$ & $1.644(1.227-2.203)$ & 0.001 & $1.592(1.111-2.280)$ & 0.011 \\
\hline Noncurative/curative treatment & $215 / 251$ & $3.412(2.538-4.587)$ & $<0.001$ & $2.789(1.932-4.026)$ & $<0.001$ \\
\hline ALBI grade 1 & 197 & 1 & & 1 & \\
\hline ALBI grade 2 & 236 & $2.672(1.926-3.706)$ & $<0.001$ & 2.279 (1.529-3.399) & $<0.001$ \\
\hline ALBI grade 3 & 33 & $7.155(4.422-11.576)$ & $<0.001$ & $7.478(4.128-13.546)$ & $<0.001$ \\
\hline
\end{tabular}

AFP, alpha-fetoprotein; ALBI, albumin-bilirubin; ALP, alkaline phosphatase; ALT, alanine aminotransferase; AST, aspartate aminotransferase; CI, confidence interval; EVs, esophageal varices; HBsAg, hepatitis B virus surface antigen; HCV, hepatitis C virus; HR, hazard ratio; PT/INR, prothrombin time/international normalized ratio; SLHCC, single large hepatocellular carcinoma.

as the selection guideline of the indication for liver transplantation in HCC patients. By meeting the Milan criteria, 5-year survival rates are $>70 \%$ and tumor recurrence rates are $<15 \%$. Therefore, the Milan criteria have been integrated into the BCLC staging system [8]. However, tumor size is not taken into account for staging in the 7th American Joint Committee on Cancer (AJCC) staging systems. This concept was based on several studies which showed that tumor size was not an independent risk factor for long-term survival and tumor recurrence after curative resection of single HCC. Instead, tumor biology and underlying liver disease were better prognostic factors than tumor size [36].

Nevertheless, most of these studies enrolled patients with well-preserved liver function who was feasible for resection. Several recent studies demonstrated that tumor size was one of the most important prognostic factors for single HCC, and the rationale for its omission in the BCLC and 7th AJCC staging system has been challenged [13-17, 38]. Consequently, tumor size is integrated into other HCC staging systems, including the Hong Kong Liver Cancer staging system with a cu-off set at $5 \mathrm{~cm}$ [39]. Our study also validated that patients with SLHCC had poorer outcomes than those in the BCLC stage A. These results confirm the crucial prognostic role of tumor size in HCC patients.

Approximately $80 \%$ of patients with HCC have cirrhosis. The degree of liver functional reserve is crucial in determining the treatment modality and affects the OS in HCC patients. Lower albumin levels imply dysfunction of liver synthesis and a higher risk of ascites formation in patients with cirrhosis. Moreover, serum albumin and bilirubin levels are the most critical factors to predict hepatic adverse events in cirrhotic patients. Recently, a new prognostic score, the ALBI score, has been validated as an objective, inexpensive, and feasible 
Fig. 7. Comparison of OS rates between patients who underwent resection surgery and TACE in the SLHCC group stratified by tumor size and ALBI grade. a All patients $(p<0.001)$. b Tumor size between 5 and $10 \mathrm{~cm}(p<0.001)$. c Tumor size $>10 \mathrm{~cm}(p=0.055)$. d ALBI grade $1(p=0.009)$. e ALBI grade 2 or $3(p<0.001)$. ALBI, albuminbilirubin; OS, overall survival; SLHCC, single large hepatocellular carcinoma; TACE, transarterial chemoembolization.
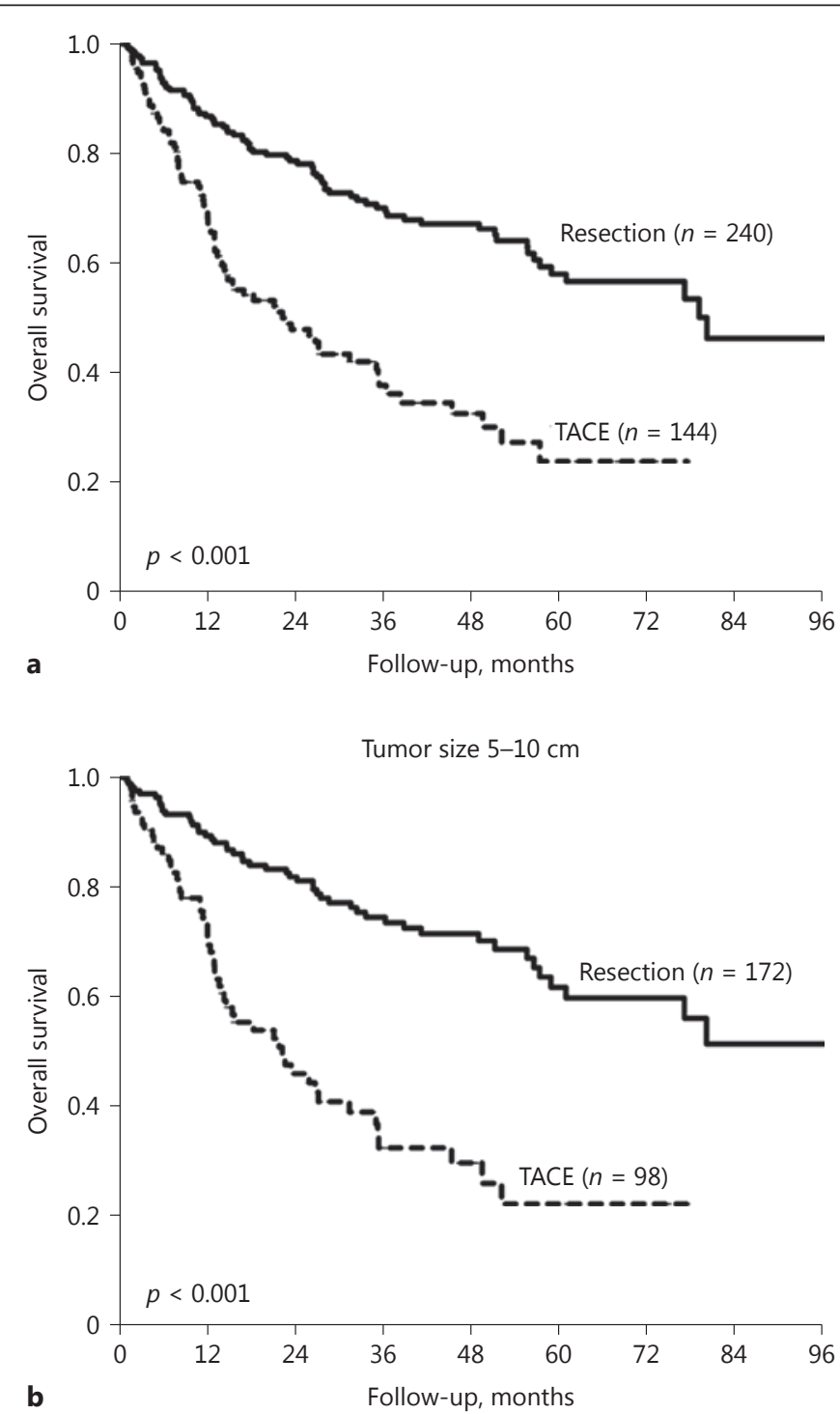

b

Tumor size $>10 \mathrm{~cm}$

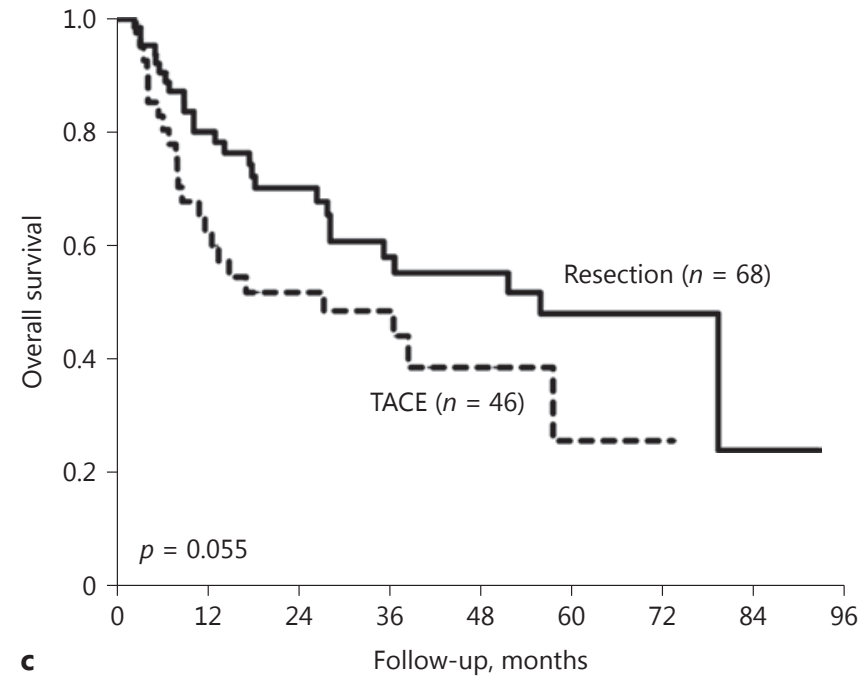


Fang et al.: Single Large Hepatocellular Carcinoma

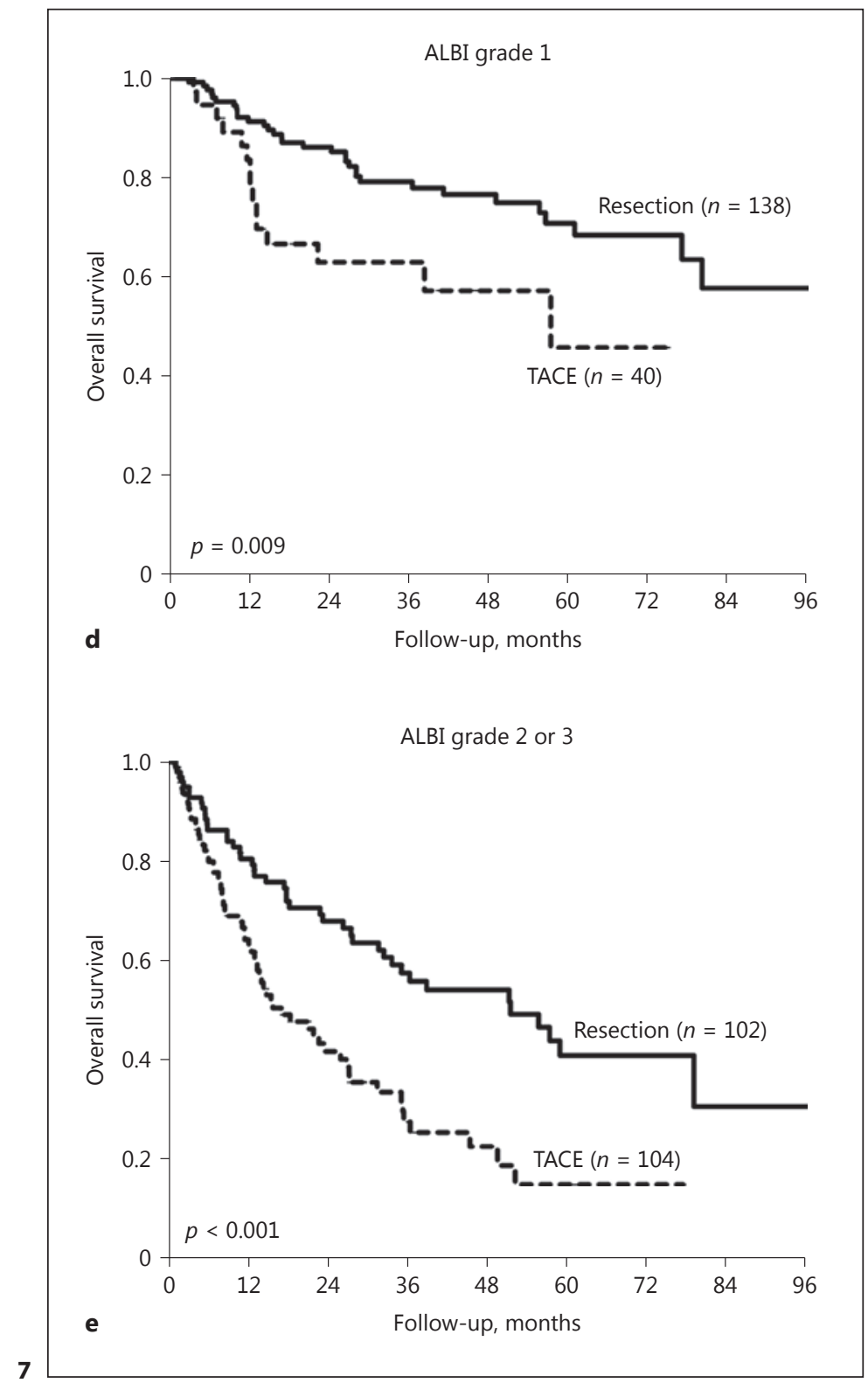

biomarker for liver function estimation and a reliable prognostic predictor across different BCLC stages and treatments for HCC [20, 21, 40-43]. In our cohort, we performed two multivariate analysis models to assess the independent risk factors predicting poor OS. In model 1 , it demonstrated that ALBI grade was correlated to poor prognosis in HCC patients. In model 2, when the ALBI grade was not enrolled, its two major determinants (serum albumin and bilirubin levels) were the independent risk factors associated with poorer OS. These results indicated that ALBI grade had an excellent discriminative ability to predict the prognoses of HCC patients.

Current HCC management guidelines recommend liver transplantation, resection surgery, and local ablation therapies as the first-line curative treatment modalities in patients with BCLC stage A HCC [8]. For patients with BCLC stage B, TACE is the recommended treatment. However, the most suitable staging and treatment for SLHCC have not yet been fully elucidated. In our study, SLHCC patients who had ALBI grade 1 had a slightly lower OS 


\section{Liver
Cancer}

\begin{tabular}{|c|c|}
\hline \multicolumn{2}{|l|}{ Liver Cancer 2018;7:335-358 } \\
\hline DOI: 10.1159/000487407 & $\begin{array}{l}\text { (c) } 2018 \text { S. Karger AG, Basel } \\
\text { www.karger.com/lic }\end{array}$ \\
\hline
\end{tabular}

Fang et al.: Single Large Hepatocellular Carcinoma

rate than those with BCLC stage A, especially those who underwent curative therapies. However, for those with poorer liver function reserve (ALBI grade 2 or 3), the prognoses were similar between patients with SLHCC and those with BCLC stage B HCC. Similarly, patients with SLHCC had an OS rate closer to BCLC stage A than BCLC stage B when they underwent curative therapy, while the prognoses were comparable between SLHCC and BCLC stage B when they received noncurative therapy. These data suggest that ALBI grade and treatment modalities could further help to stratify SLHCC into different prognostic groups. In brief, the prognoses of patients with SLHCC will be close to those with BCLC stage A when they have a relatively well-preserved liver function feasible for curative therapy, while their outcomes will be similar to those of patients in BCLC stage B when they have ALBI grade 2 or 3.

Regarding treatment, several previous studies have showed that patients with SLHCC who underwent resection had better prognoses than those who underwent TACE [44-46]. However, these results might have some biases due to several important confounding factors that affect prognoses. These include liver functional reserves, which existed in patients who underwent resection and TACE. In the present cohort, treatment modality was still an important prognostic factor for patients with SLHCC after adjusting for the confounding factors by multivariate analysis and subgroup analysis stratified by tumor size and liver functional reserve. It is suggested that curative treatment, especially resection surgery, could serve as a first-line treatment modality for SLHCC if it is not contraindicated.

There are some limitations to this study. First, we included HCC patients from a single tertiary center. The data should be validated externally in other regions of the world in which the selection of treatment modalities, the quality of technical skills, and the supportive systems for patients might vary substantially. Second, interobserver bias may exist in the amount of ascites and the degree of hepatic encephalopathy for Child-Pugh scoring. This is an important parameter of the BCLC staging system. Third, this is a retrospective study, and the choice of curative or noncurative treatment was primarily based on the physician's judgment and recommendation for a substantial number of patients with SLHCC. Thus, selection bias could not be completely avoided. Although the long-term prognoses of patients undergoing different therapies were not all equal, we categorized treatment modalities as curative and noncurative therapies due to the relatively small number of patients treated with some therapies, such as liver transplantation and radiofrequency ablation in the SLHCC group. Nevertheless, we compared the outcomes between resection surgery and TACE, the most common curative and noncurative treatments for SLHCC, respectively. It was shown that resection surgery could provide a survival benefit compared with TACE in most of the subgroups, except in the setting of tumor size $>10 \mathrm{~cm}(p=0.055)$, which might be due to the small number of patients in this very large tumor group. Further large-scale studies are warranted to compare the prognosis between resection surgery and TACE for patients with a very large solitary HCC. Fourth, since among the patients with an ALBI grade 3 in the SLHCC group, only 7 and 10 patients underwent resection surgery and TACE, respectively, we could not assess the long-term prognoses between resection and TACE in such patients. Further prospective studies are needed to elucidate this issue. Fifth, there were substantial differences in demographic characteristics, especially liver functional reserve, among patients in the three groups. Confounding differences might make evaluating the true impact of tumor size on posttreatment prognoses difficult.

In conclusion, patients with SLHCC are suggested to be assigned to an independent group, different from BCLC stage A and B. ALBI grade could help to stratify SLHCC into different prognostic subgroups. In addition, curative treatment modality provided a better prognosis than noncurative therapy, regardless of tumor stage. 
Fang et al.: Single Large Hepatocellular Carcinoma

\section{Acknowledgment}

Writing assistance: American Manuscript Editors.

\section{Statement of Ethics}

The study complied with the standards of the Declaration of Helsinki as well as current ethical guidelines. It was approved by the Institutional Review Board of Taipei Veterans General Hospital (VGHIRB No. 2017-07-022BC). Consent waivers were obtained, and patient information and records were anonymized and de-identified prior to analysis.

\section{Disclosure Statement}

There is no potential conflict of interest involved in this paper.

\section{Funding Sources}

This study was supported by grants from the Ministry of Science and Technology of Taiwan (MOST 104-2314-B-075-07, 105-2314-B-075-041, 106-2314-B-075-043), Taipei Veterans General Hospital (V106C122, Center of Excellence for Cancer Research MOHW106-TDU-B-211-144-003), the Taipei Veterans General Hospital-National Taiwan University Hospital Joint Research Program (TVGH-NTUH VN106-03), and the Taipei Veterans General Hospital-National Yang-Ming University Excellent Physician Scientists Cultivation Program (106-V-B-029).

\section{References}

1 Ferlay J, Soerjomataram I, Dikshit R, Eser S, Mathers C, Rebelo M, et al: Cancer incidence and mortality worldwide: sources, methods and major patterns in GLOBOCAN 2012. Int J Cancer 2015;136:E359-E386.

-2 Kudo M, Izumi N, Sakamoto M, Matsuyama Y, Ichida T, Nakashima O, et al: Survival analysis over 28 years of 173,378 patients with hepatocellular carcinoma in Japan. Liver Cancer 2016;5:190-197.

-3 Singal AG, El-Serag HB: Hepatocellular carcinoma from epidemiology to prevention: translating knowledge into practice. Clin Gastroenterol Hepatol 2015;13:2140-2151.

4 Kudo M: Surveillance, diagnosis, treatment, and outcome of liver cancer in Japan. Liver Cancer 2015;4:39-50.

5 Siegel RL, Miller KD, Jemal A: Cancer statistics, 2015. CA Cancer J Clin 2015;65:5-29.

6 Bruix J, Sherman M, Llovet JM, Beaugrand M, Lencioni R, Burroughs AK, et al: Clinical management of hepatocellular carcinoma. Conclusions of the Barcelona-2000 EASL conference. European Association for the Study of the Liver. J Hepatol 2001;35:421-430.

7 Kao WY, Chao Y, Chang CC, Li CP, Su CW, Huo TI, et al: Prognosis of early-stage hepatocellular carcinoma: the clinical implications of substages of Barcelona Clinic Liver Cancer system based on a cohort of 1,265 patients. Medicine (Baltimore) 2015;94:e1929.

8 Bruix J, Sherman M: Management of hepatocellular carcinoma: an update. Hepatology 2011;53:1020-1022.

9 Chang WT, Kao WY, Chau GY, Su CW, Lei HJ, Wu JC, et al: Hepatic resection can provide long-term survival of patients with non-early-stage hepatocellular carcinoma: extending the indication for resection? Surgery 2012;152:809-820.

10 Llovet JM, Bru C, Bruix J: Prognosis of hepatocellular carcinoma: the BCLC staging classification. Semin Liver Dis 1999;19:329-338.

11 Bruix J, Llovet JM: Prognostic prediction and treatment strategy in hepatocellular carcinoma. Hepatology 2002;35:519-524.

12 Forner A, Reig ME, de Lope CR, Bruix J: Current strategy for staging and treatment: the BCLC update and future prospects. Semin Liver Dis 2010;30:61-74.

13 Hwang S, Lee YJ, Kim KH, Ahn CS, Moon DB, Ha TY, et al: The impact of tumor size on long-term survival outcomes after resection of solitary hepatocellular carcinoma: single-institution experience with 2,558 patients. J Gastrointest Surg 2015;19:1281-1290.

14 Zhong JH, Pan LH, Wang YY, Cucchetti A, Yang T, You XM, et al: Optimizing stage of single large hepatocellular carcinoma: a study with subgroup analysis by tumor diameter. Medicine 2017;96:e6608. 


\section{Liver Cancer}

\begin{tabular}{l|l}
\hline Liver Cancer 2018;7:335-358 \\
\hline DOI: 10.1159/000487407 & $\begin{array}{l}\text { @ 2018 S. Karger AG, Basel } \\
\text { www.karger.com/lic }\end{array}$ \\
\hline
\end{tabular}

Fang et al.: Single Large Hepatocellular Carcinoma

15 Liu PH, Su CW, Hsu CY, Hsia CY, Lee YH, Huang YH, et al: Solitary large hepatocellular carcinoma: staging and treatment strategy. PLoS One 2016;11:e0155588.

16 Jung YK, Jung CH, Seo YS, Kim JH, Kim TH, Yoo YJ, et al: BCLC stage B is a better designation for single large hepatocellular carcinoma than BCLC stage A. J Gastroenterol Hepatol 2016;31:467-474.

17 Cho Y, Sinn DH, Yu SJ, Gwak GY, Kim JH, Yoo YJ, et al: Survival analysis of single large (>5 cm) hepatocellular carcinoma patients: BCLC A versus B. PLoS One 2016;11:e0165722.

18 Torzilli G, Belghiti J, Kokudo N, Takayama T, Capussotti L, Nuzzo G, et al: Reply to letter: “Dissecting EASL/ AASLD recommendations with a more careful knife: a comment on 'surgical misinterpretation' of the BCLC staging system": real misinterpretation or lack of clarity within the BCLC? Ann Surg 2015;262:e18-e19.

19 Hsieh WY, Chen PH, Lin IY, Su CW, Chao Y, Huo TI, et al: The impact of esophagogastric varices on the prognosis of patients with hepatocellular carcinoma. Sci Rep 2017;7:42577.

20 Kao WY, Su CW, Chiou YY, Chiu NC, Liu CA, Fang KC, et al: Hepatocellular carcinoma: nomograms based on the albumin-bilirubin grade to assess the outcomes of radiofrequency ablation. Radiology 2017;285:670-680.

-21 Johnson PJ, Berhane S, Kagebayashi C, Satomura S, Teng M, Reeves HL, et al: Assessment of liver function in patients with hepatocellular carcinoma: a new evidence-based approach - the ALBI grade. J Clin Oncol 2015; 33:550-558

22 Hiraoka A, Kumada T, Kudo M, Hirooka M, Tsuji K, Itobayashi E, et al: Albumin-bilirubin (ALBI) grade as part of the evidence-based clinical practice guideline for HCC of the Japan Society of Hepatology: a comparison with the liver damage and Child-Pugh classifications. Liver Cancer 2017;6:204-215.

23 Bruix J, Sherman M: Management of hepatocellular carcinoma. Hepatology 2005;42:1208-1236.

24 Kao WY, Chiou YY, Hung HH, Su CW, Chou YH, Huo TI, et al: Younger hepatocellular carcinoma patients have better prognosis after percutaneous radiofrequency ablation therapy. J Clin Gastroenterol 2012;46:62-70.

25 Hsu CY, Lee YH, Hsia CY, Huang YH, Su CW, Lin HC, et al: Performance status in patients with hepatocellular carcinoma: determinants, prognostic impact, and ability to improve the Barcelona Clinic Liver Cancer system. Hepatology 2013;57:112-119.

26 Hung HH, Lei HJ, Chau GY, Su CW, Hsia CY, Kao WY, et al: Milan criteria, multi-nodularity, and microvascular invasion predict the recurrence patterns of hepatocellular carcinoma after resection. J Gastrointest Surg 2013; 17:702-711.

-27 Kao WY, Chiou YY, Hung HH, Su CW, Chou YH, Wu JC, et al: Serum alpha-fetoprotein response can predict prognosis in hepatocellular carcinoma patients undergoing radiofrequency ablation therapy. Clin Radiol 2012;67: 429-436.

-28 Berzigotti A, Reig M, Abraldes JG, Bosch J, Bruix J: Portal hypertension and the outcome of surgery for hepatocellular carcinoma in compensated cirrhosis: a systematic review and meta-analysis. Hepatology 2015;61: 526-536.

-29 Fang KC, Su CW, Chiou YY, Lee PC, Chiu NC, Liu CA, et al: The impact of clinically significant portal hypertension on the prognosis of patients with hepatocellular carcinoma after radiofrequency ablation: a propensity score matching analysis. Eur Radiol 2017;27:2600-2609.

30 Wu JC, Huang YH, Chau GY, Su CW, Lai CR, Lee PC, et al: Risk factors for early and late recurrence in hepatitis B-related hepatocellular carcinoma. J Hepatol 2009;51:890-897.

-31 Su CW, Chau GY, Hung HH, Yeh YC, Lei HJ, Hsia CY, et al: Impact of steatosis on prognosis of patients with earlystage hepatocellular carcinoma after hepatic resection. Ann Surg Oncol 2015;22:2253-2261.

-32 Su CW, Chiou YW, Tsai YH, Teng RD, Chau GY, Lei HJ, et al: The influence of hepatitis B viral load and pre-S deletion mutations on post-operative recurrence of hepatocellular carcinoma and the tertiary preventive effects by anti-viral therapy. PLoS One 2013;8:e66457.

-33 Cucchetti A, Djulbegovic B, Tsalatsanis A, Vitale A, Hozo I, Piscaglia F, et al: When to perform hepatic resection for intermediate-stage hepatocellular carcinoma. Hepatology 2015;61:905-914.

-34 Hung HH, Chiou YY, Hsia CY, Su CW, Chou YH, Chiang JH, et al: Survival rates are comparable after radiofrequency ablation or surgery in patients with small hepatocellular carcinomas. Clin Gastroenterol Hepatol 2011; 9:79-86.

-35 D’Avola D, Inarrairaegui M, Pardo F, Rotellar F, Marti P, Bilbao JI, et al: Prognosis of hepatocellular carcinoma in relation to treatment across BCLC stages. Ann Surg Oncol 2011;18:1964-1971.

-36 Kluger MD, Salceda JA, Laurent A, Tayar C, Duvoux C, Decaens T, et al: Liver resection for hepatocellular carcinoma in 313 Western patients: tumor biology and underlying liver rather than tumor size drive prognosis. J Hepatol 2015;62:1131-1140.

-37 Mazzaferro V, Regalia E, Doci R, Andreola S, Pulvirenti A, Bozzetti F, et al: Liver transplantation for the treatment of small hepatocellular carcinomas in patients with cirrhosis. N Engl J Med 1996;334:693-699.

38 Chan AC, Fan ST, Poon RT, Cheung TT, Chok KS, Chan SC, et al: Evaluation of the seventh edition of the American Joint Committee on Cancer tumour-node-metastasis (TNM) staging system for patients undergoing curative resection of hepatocellular carcinoma: implications for the development of a refined staging system. HPB (Oxford) 2013;15:439-448.

-39 Yau T, Tang VY, Yao TJ, Fan ST, Lo CM, Poon RT: Development of Hong Kong Liver Cancer staging system with treatment stratification for patients with hepatocellular carcinoma. Gastroenterology 2014;146:16911700.e3.

40 Waked I, Berhane S, Toyoda H, Chan SL, Stern N, Palmer D, et al: Transarterial chemo-embolisation of hepatocellular carcinoma: impact of liver function and vascular invasion. Br J Cancer 2017;116:448-454. 
41 Toyoda H, Lai PB, O’Beirne J, Chong CC, Berhane S, Reeves H, et al: Long-term impact of liver function on curative therapy for hepatocellular carcinoma: application of the ALBI grade. Br J Cancer 2016;114:744-750.

42 Pinato DJ, Sharma R, Allara E, Yen C, Arizumi T, Kubota K, et al: The ALBI grade provides objective hepatic reserve estimation across each BCLC stage of hepatocellular carcinoma. J Hepatol 2017;66:338-346.

43 Lee PC, Chen YT, Chao Y, Huo TI, Li CP, Su CW, et al: Validation of the albumin-bilirubin grade-based integrated model as a predictor for sorafenib-failed hepatocellular carcinoma. Liver Int 2018;38:321-330.

-44 Stevens CL, Awad A, Abbas SM, Watters DAK: Systematic review and meta-analysis of hepatic resection versus transarterial chemoembolization for solitary large hepatocellular carcinoma. HPB (Oxford) 2017;19:653658.

45 Zhong JH, Ke Y, Gong WF, Xiang BD, Ma L, Ye XP, et al: Hepatic resection associated with good survival for selected patients with intermediate and advanced-stage hepatocellular carcinoma. Ann Surg 2014;260:329340.

46 Ho MC, Hasegawa K, Chen XP, Nagano H, Lee YJ, Chau GY, et al: Surgery for intermediate and advanced hepatocellular carcinoma: a consensus report from the 5th Asia-Pacific Primary Liver Cancer Expert Meeting (APPLE 2014). Liver Cancer 2016;5:245-256. 